\title{
Locally Accurate Tensor Networks for Thermal States and Time Evolution
}

\author{
Álvaro M. Alhambra $\circledast^{1,2, *}$ and J. Ignacio Cirac ${ }^{1,2}$ \\ ${ }^{1}$ Max-Planck-Institut für Quantenoptik, Hans-Kopfermann-Straße 1, Garching D-85748, Germany \\ ${ }^{2}$ Munich Center for Quantum Science and Technology (MCQST), Schellingstr. 4, München D-80799, Germany
}

(Received 17 June 2021; accepted 20 October 2021; published 10 November 2021)

\begin{abstract}
Tensor-network methods are routinely used in approximating various equilibrium and nonequilibrium scenarios, with the algorithms requiring a small bond dimension at low enough time or inverse temperature. These approaches so far lacked a rigorous mathematical justification, since existing approximations to thermal states and time evolution demand a bond dimension growing with system size. To address this problem, we construct projected entangled-pair operators that approximate, for all local observables, (i) their thermal expectation values and (ii) their Heisenberg time evolution. The bond dimension required does not depend on system size, but only on the temperature or time. We also show how these can be used to approximate thermal correlation functions and expectation values in quantum quenches.
\end{abstract}

DOI: 10.1103/PRXQuantum.2.040331

\section{INTRODUCTION}

The classical simulation of quantum many-body systems is an important challenge for many different fields, including condensed-matter physics, quantum chemistry, quantum information, and high-energy physics. Approximating generic settings efficiently is widely believed to be impossible, due to the exponential growth of the Hilbertspace dimension with the system size. However, many situations of interest do not occur on generic regions of the Hilbert space, but are rather confined to the "physical corner" of it. This can then be covered by appropriate variational ansätze, with tensor networks being the most prominent example.

Indeed, tensor-network methods based on the densitymatrix renormalization group (DMRG) algorithm [1] are routinely used for the simulation of many important physical situations. Most prominently, they are used for lowenergy properties in one and even two dimensions, with great success [2]. They are also widely used in the approximation of finite temperature phenomena [3-13], and in the simulation of dynamics [8,14-26] for short times. This allows for the computation of properties on large system sizes in many situations of interest.

These methods are supported by a series of mathematically rigorous results. For low energies, it is known that

*alvaro.alhambra@mpq.mpg.de

Published by the American Physical Society under the terms of the Creative Commons Attribution 4.0 International license. Further distribution of this work must maintain attribution to the author(s) and the published article's title, journal citation, and DOI. ground states of gapped models in one dimension have good matrix-product-state (MPS) approximations [27-29], and that these approximations can be found efficiently with explicit algorithms [30-32] (see Refs. [33,34] for current progress in two dimensions). For thermal states $\propto e^{-\beta H}$, it is known that they can be approximated in any dimension by tensor networks if $\beta$ is not too large [35-38]. Similar results are also known for the unitary time evolution at short times $e^{-i t H}$ [38-40]. All of these previous works aim at approximating the whole ground state, thermal state, or unitary, respectively. This can be achieved with a bond dimension that grows with system size.

However, for many physical applications, such as calculating local order parameters, one does not necessarily require a full global approximation, but just a tensor network that describes the relevant local properties well. The success of existing numerical implementations suggests that a much smaller bond dimension, independent of system size, is required in this case.

This problem has been previously explored for ground states: that such local approximations exist in onedimensional (1D) gapped models has been shown in a mathematically rigorous way. First, with matrix product operators (MPOs) [41,42] and more recently with MPS [43-45], as well as with projected entangled pairs (PEPS) for two-dimensional (2D) ground states with an area law $[44,45]$ (see Ref. [46] for a perspective). For thermal states and time evolution, previous results indicate that it is possible to simulate specific local properties in an efficient way $[37,40]$. However, it was previously not known whether there exist particular tensor networks that approximate all the local properties of a system with a bond dimension independent of system size. 
Here we address this question, by constructing tensor networks with a provably small bond dimension that approximate, for any local operator $A$ and in any spatial dimension.

(a) Thermal expectation values $\langle A\rangle_{\beta} \equiv \operatorname{Tr}\left[A\left(e^{-\beta H} / Z\right)\right]$.

(b) The Heisenberg time evolution $e^{-i t H} A e^{i t H}$.

By linearity, they also approximate extensive sums of local observables $A=(1 / N) \sum_{x=0}^{N} A_{x}$. The results hold for Hamiltonians $H$ that are short ranged, but not necessarily translation invariant. The bond dimension has a similar dependence on $\beta$ and $t$ as previous global approximations, but it now does not grow with system size.

Notably, our constructions are explicit, and give rise to algorithms that can, in principle, be implemented in practice. While these are likely less efficient or more cumbersome to implement than other known methods used in practice, the advantage is that we have performance guarantees. These do not currently exist for most algorithms used in practice, such as the paradigmatic DMRG algorithm. Theoretical guarantees for certain algorithms support the fact that state-of-the-art methods give accurate results. This is because said guarantees show that the methods target quantities that can, in principle, be computed efficiently.

We prove these guarantees with the aid of previous results on global approximations [35-39], combined with ideas that allow us to exploit the locality of the problem. For thermal states, this is the principle usually known as the local indistinguishability [37,47-49], which relies on the clustering of correlations [49,50]. For time evolution it is the Lieb-Robinson bound [51,52]. We also introduce a tensor-network construction of a linear map that outputs different projected entangled-pair operator (PEPO) approximations for the unitary dynamics of local operators depending on their support, which may be of independent interest.

We then show how these results allow us to compute quantities of interest. We focus on approximations to autocorrelation functions (such as current operators in transport problems [53-59]), and time-dependent local expectation values in quantum quenches [21,25,60-62]. These two are particularly relevant to current experiments in quantum-simulation platforms, such as cold atoms, superconducting qubits, or trapped ions, since they are some of the most easily measurable and informative quantities.

The paper is structured as follows. First, we explain the definitions and the setting in Sec. II. Then, we show our result for local thermal states in Sec. III, and for time evolution in Sec. IV. We explain the impact of our results for correlation functions and quantum quenches in Sec. V, and conclude. The technical proofs and further background are placed in the Appendices.

\section{SETTING AND DEFINITIONS}

Throughout this work, the notions of approximation used are in terms of closeness in 1-norm or trace norm $\|X\|_{1}$ for quantum states and their PEPO approximations [63], and the operator norm $\|X\|$ for operators. The big- $\mathcal{O}$ notation indicates that a quantity $f=\mathcal{O}(n)$ is such that for some constant $c, f \leq c n$. $\tilde{\mathcal{O}}(n)$ indicates polylogarithmic corrections $f \leq c n \times \operatorname{polylog}(n)$, and $o(n)$ that the scaling is strictly smaller than linear in $n$.

We focus on systems governed by a local Hamiltonian $H=\sum_{x} h_{x}$ with uniformly bounded, short-range interactions $\max _{x}\left\|h_{x}\right\| \leq h$ between $N$ particles of small local dimension. The interactions have the graph structure of a $d$-dimensional lattice $\Lambda$ with growth constant $\gamma$ and maximum degree $z$. We denote the small connected regions we focus on as $\mathcal{R}$, which have a maximum length $k$, such that $|\mathcal{R}| \leq k^{d}$ (that is, the small region can be embedded on a hypercube of length $k$ ).

The operators that approximate $e^{-i t H}$ and $e^{-\beta H}$ are MPOs and their higher-dimensional generalization PEPOs [64], which are operator generalizations of MPS and PEPS, respectively. In $d=1$, an MPO $M_{D}$ of bond dimension $D$ can be written simply as $[3,64,65]$

$$
\begin{aligned}
M_{D}= & \sum_{\substack{s_{1}, s_{2}, \ldots, s_{n}=1 \\
s_{1}^{\prime}, s_{2}^{\prime}, \ldots, s_{n}^{\prime}=1}}^{N} \operatorname{Tr}\left[B_{1}^{\left[s_{1}, s_{1}^{\prime}\right]} B_{2}^{\left[s_{2}, s_{2}^{\prime}\right]} \cdots B_{n}^{\left[S_{n}, s_{n}^{\prime}\right]}\right] \\
& \times\left|s_{1}, s_{2}, \ldots, s_{n}\right\rangle\left\langle s_{1}^{\prime}, s_{2}^{\prime}, \ldots, s_{n}^{\prime}\right|,
\end{aligned}
$$

where each of the matrices $\left\{B_{i}^{\left[s_{i}, s_{i}^{\prime}\right]}\right\}_{i, s_{i}, s_{i}^{\prime}}$ is of dimension $D \times D$.

On the other hand, PEPOs are defined in terms of the interaction graph with edges $\{e\} \in \mathcal{E}$ and vertices $\{v\} \in \mathcal{V}$ as $[36,64,66]$

$$
M_{D}=\sum_{\alpha: \mathcal{E} \rightarrow\{1, \ldots, D\}} \bigotimes_{v \in \mathcal{V}} X_{\alpha\left(e_{1}^{v}\right), \ldots, \alpha\left[e_{z}^{v}(v)\right]}^{v}
$$

where $X_{\alpha\left(e_{1}^{v}\right), \ldots, \alpha\left[e_{z}^{v}(v)\right]}^{v}$ is an operator acting on vertex $v$, $z(v)$ is its degree and $e_{1}^{v}, \ldots, e_{z}^{v}(v)$ are the vertices going through it. See Refs. [36,64] for more detailed descriptions.

We also introduce the notion of a PEPO map, which appears in one of our main results (Result 3). This is a linear map $\mathcal{M}(A)$ that takes a PEPO $A$ as an input, and outputs another PEPO with a potentially larger bond dimension. Schematically, it can be understood as 


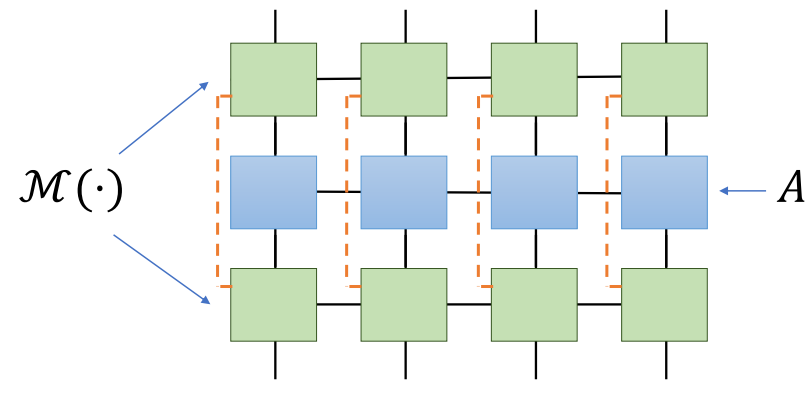

That is, it can be written as a product of PEPOs acting on each of the two physical indices of $A$, and with an additional "physical" index (in the figure, orange and dashed) that is contracted, such that $\mathcal{M}(A)=\sum_{i} M_{D}^{(i)} A\left(M_{D}^{(i)}\right)^{\dagger}$ for some PEPOs $M_{D}^{(i)}$.

\section{LOCAL APPROXIMATIONS TO THERMAL STATES}

We start with local approximations to thermal states. Let $\mathcal{R}$ be a small region as defined in Sec. II. The local thermal state is then $\operatorname{Tr}_{\Lambda \backslash \mathcal{R}}\left[e^{-\beta H} / Z\right]$, and the marginal of the PEPO approximation $\tilde{\rho}_{k}$ is $\operatorname{Tr}_{\Lambda \backslash \mathcal{R}}\left[\tilde{\rho}_{k}\right]$.

A first idea to approximate the marginals could be to simply consider a product $\bigotimes_{i} \operatorname{Tr}_{\Lambda \backslash \mathcal{R}_{i}}\left[e^{-\beta H} / Z\right]$ for some choice of adjacent regions $\left\{\mathcal{R}_{i}\right\}$. However, this clearly yields a large error in the regions that lie within two adjacent $\mathcal{R}_{i}$. Since we want to approximate all of them at once, we need a scheme that has no preferred partition of the lattice $\left\{\mathcal{R}_{i}\right\}$. This is possible with a uniform average over large enough partitions, and aided by local indistinguishability, which states that the marginal of large thermal states can be approximated by the marginals of much smaller thermal states (see Lemma 1 for the precise statement).

One key assumption we need is the decay of correlations

$$
\max _{X, Y} \frac{\left|\langle X \otimes Y\rangle_{\beta}-\langle X\rangle_{\beta}\langle Y\rangle_{\beta}\right|}{\|X\||| Y||} \leq \varepsilon(l)
$$

where the optimization is over arbitrary observables separated by a distance $l$ on the lattice, and $\varepsilon(l)$ is a function decreasing with $l$.

The most prominent decay is exponential $\varepsilon(l) \leq e^{-l / \xi}$, which defines the thermal correlation length $\xi$. This has been proven for translation invariant chains [50,67], and there is strong evidence that it holds in any 1D thermal state $[50,68]$. In higher dimensions it holds only above a finite threshold temperature $\beta^{*}$, as shown in Refs. [37,69], which also give a bound on the correlation length. However, we can also consider the polynomial decay $\varepsilon(l) \leq$ $R / l^{d+1}$ for some constant $R>0$. This might correspond to the behavior at certain thermal phase transitions.
The decay of correlations is required to simulate local properties without a system size dependence, since one needs to be able to isolate them from distant regions. This is only possible if the correlations decay sufficiently fast, so that regions of width approximately $\mathcal{O}(\xi)$ can be approximated independently of the rest.

With this, we now show the main result of this section.

Result 1. There is an explicit construction of a PEPO $\tilde{\rho}_{k}$ such that for any region $\mathcal{R}$ on the lattice $\Lambda$ it locally approximates the thermal state

$$
\left\|\operatorname{Tr}_{\Lambda \backslash \mathcal{R}}\left[\frac{e^{-\beta H}}{Z}\right]-\operatorname{Tr}_{\Lambda \backslash \mathcal{R}}\left[\tilde{\rho}_{k}\right]\right\|_{1} \leq \epsilon
$$

The bond dimension is bounded as follows.

(a) For $d=1$, assuming correlations decay exponentially, it is a MPO with bond dimension

$$
\begin{aligned}
D \leq & \left(\frac{k+\xi}{\epsilon}\right) \\
& \times \exp \left(\tilde{\mathcal{O}}\left\{\max \left[\beta, \sqrt{\beta \log \left(\frac{k+\xi}{\epsilon^{2}}\right)}\right]\right\}\right),
\end{aligned}
$$

which is quasilinear in $(k+\xi) / \epsilon$ for any $\beta \simeq \mathcal{O}(1)$.

(b) In higher dimensions $d>1$ and at high temperature $\beta \leq \beta^{*} \equiv \log [(1+\sqrt{1+4 / \gamma}) / 2] / 2 h$ the bond dimension is

$$
D \leq\left(C^{\prime} \beta d \max \left\{\frac{k^{d}}{\epsilon^{2}}, \frac{d^{2 d} \xi(\beta)^{d^{2}}}{\epsilon^{d+1}}\right\}\right)^{\mathcal{O}(\beta d)},
$$

where $C^{\prime}$ is constant and the correlation length is $\xi(\beta) \equiv\left|\left\{\log \left[\gamma e^{2 \beta h}\left(e^{2 \beta h}-1\right)\right]\right\}^{-1}\right|$.

(c) For lower temperatures, if the correlations decay polynomially $\varepsilon(l) \leq R / l^{d+1}, D$ is bounded as in Eqs. (5) and (6) but replacing $\xi^{d}$ with $R$.

The bond dimension in both cases grows with $k, \xi, \beta$, and $\epsilon^{-1}$, as expected. This result implies a good approximation in any local expectation value, since $\| \operatorname{Tr}_{\Lambda \backslash \mathcal{R}}[\rho]-$ $\operatorname{Tr}_{\Lambda \backslash \mathcal{R}}[\sigma] \|_{1}=\max _{\operatorname{supp}(A) \in \mathcal{R},\|A\|=1} \operatorname{Tr}[A(\rho-\sigma)]$. See Fig. 1 for an illustration.

The proof is shown in Appendix B. It draws inspiration from previous results on local approximations of pure states [41-44]. The PEPO here is the uniform average of tensor products of approximations to local thermal states. These lie on consecutive hypercubes of a given size $l_{0}^{d}$, which span the whole lattice. The average is taken over all the different $l_{0}^{d}$ partitions of the lattice (that is, the different displacements of a given partition into hypercubes, see Fig. 4). 


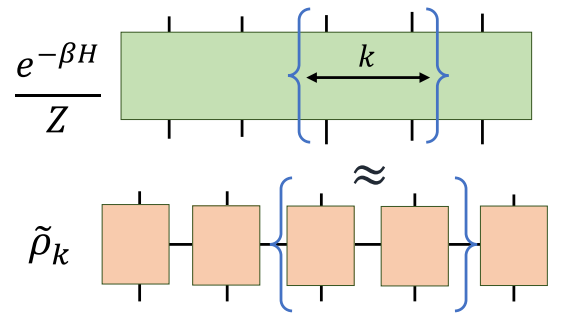

FIG. 1. Schematic illustration of Result 1, where we approximate subsystems of a thermal state. $\tilde{\rho}_{k}$ is the local PEPO approximation, and the regions approximated are of length $k$.

Each of the PEPOs on the hypercubes approximates $\operatorname{Tr}_{\Lambda \backslash \mathcal{R}}\left[e^{-\beta H} / Z\right]$ well for a given partition provided that $\mathcal{R}$ is far from the boundary between adjacent hypercubes. That this happens for most partitions is guaranteed by a result from Ref. [49], which shows that any local marginal of a thermal state does not depend on the regions far away from it (here how "far away" is determined by the thermal correlation length). This is exactly the idea behind local indistinguishability [49], and the related concept of locality of temperature [37,70-73], which states that (i) subsystems of thermal states of quantum Hamiltonians are robust to distant perturbations and that (ii) they are close to the marginals of the thermal state of their vicinity (see Ref. [37] for further discussions on this idea).

The smaller PEPOs within the hypercubes can then be taken to be any of the existing global approximations to thermal states. The current best estimates are given in Ref. [38] for $1 \mathrm{D}$, which is $D \leq \exp \left[\mathcal{O}\left(\sqrt{\beta \log \left(l_{0} / \epsilon\right)}\right)\right]$ and in Ref. [36] for higher dimensions, which is $D \leq$ $\left(\beta l_{0}^{d} / \epsilon\right)^{\mathcal{O}(\beta d)}$. In the proof, one has to choose $l_{0}$ small to keep the bond dimension controlled, but still large enough such that the error from the local indistinguishability estimate is $\mathcal{O}(\epsilon)$. This leads to Eq. (5) [by choosing $l_{0} \propto(k+\xi) / \epsilon$ ] and Eq. (6) [by choosing $l_{0} \propto$ $\left.\max \left\{k \epsilon^{-d}, d \xi^{d} / \epsilon\right\}\right]$.

Let us comment on the algorithmic implications of Result 1. To build the MPO and PEPO here one just needs to construct them as given by the prescriptions of Refs. [36,38], respectively. In Ref. [38], the onedimensional approximation is defined as a product of the Taylor expansion of operators $e^{-\beta H_{j}}$, where $H_{j}$ is the Hamiltonian in a small region. Similar algorithms have already appeared in the literature [10,11]. In Ref. [36], the higher-dimensional approximation is based on the linked cluster expansion [35], which can in principle also be implemented numerically [26,74]. Standard MPO and PEPO results $[2,64]$ guarantee that these approximations can be computed via an algorithm with run-time poly $(D, N)$.

\section{LOCAL APPROXIMATIONS TO TIME EVOLUTION}

We now focus on efficient approximations to the Heisenberg time evolution $e^{-i t H} A e^{i t H}$. The existing results on global approximations [36-39] show that the bond dimension of a PEPO $M^{t}$ that approximates $\left\|e^{-i t H}-M^{t}\right\| \leq \epsilon$ must grow with system size. We again drop this dependence when our target is the Heisenberg evolution of local operators. The key idea is to use the Lieb-Robinson bound [51], which states that the evolution $e^{-i t H} A e^{i t H}$ is restricted to a certain "light cone" much smaller than the whole system. The evolution in this light cone can be thought of as being generated by the Hamiltonian $H$ restricted to the vicinity of $A$.

To simulate this evolution, the first idea could be to simply reduce the problem to simulating the Lieb-Robinson light cone exactly, which requires only a unitary in a region of size $\propto \mathcal{O}\left[v_{\mathrm{LR}} t+\log \left(\epsilon^{-1}\right)\right]$, where $v_{\mathrm{LR}}$ is the Lieb-Robinson velocity. While this can be done with a bond dimension independent of system size, as previously pointed in Ref. [40], it would only be a good approximation for observables in a specific small region.

Here we show how the idea of using the effective light cones of the evolution can be pushed further in order to build a single tensor network that approximates the LiebRobinson light cone of any local operator. The statements for one and higher dimensions differ significantly, and are presented separately.

\section{A. One dimension}

In one dimension, our main result essentially shows that the previous global approximation scheme from Ref. [39] simulates well any Lieb-Robinson light cone, and that in order to do so one requires only a bond dimension depending on the size of the light cone, and not on $N$. The result is as follows.

Result 2. For any operator $A$ with support on a small region $\mathcal{R}$, the Heisenberg time evolution is well approximated as

$$
\left\|e^{-i t H} A e^{i t H}-M_{k}^{t} A\left(M^{t}\right)_{k}^{\dagger}\right\| \leq 3 \epsilon\|A\|,
$$

where $M_{k}^{t}$ is a MPO with bond dimension

$$
D \leq e^{\mathcal{O}(|t|)} \times \text { poly }\left[\frac{k+v_{L R} t+\log (1 / \epsilon)}{\epsilon}\right] .
$$

That is, the bond dimension scales polynomially in $k, \epsilon^{-1}$ with a constant degree, and exponentially in time, which is consistent with the expected linear growth in entanglement along a generic time evolution [75-77]. The numerator in the polynomial of Eq. (8) corresponds to the size of the light cone that needs to be approximated. See Fig. 2 for an illustration. 


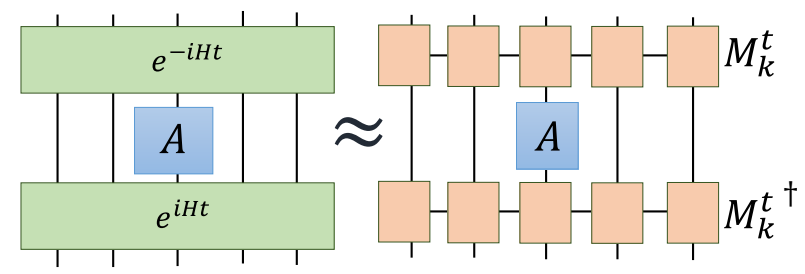

FIG. 2. Schematic illustration of Result 2, where we approximate the Heisenberg evolution $e^{-i H t} A e^{i H t}$ within the effective light cone. The operator $A$ can have support on at most $k$ adjacent sites.

The proof is shown in Appendix C 1. The construction is the same as that of Ref. [39], which shows that $e^{-i t H}$ can be approximated by a quantum circuit of depth 2 , in which the size of the gates grows with $t, \epsilon^{-1}$ and system size $N$. To drop the system size dependence we give an argument based on the Lieb-Robinson bound, which shows that to simulate the light cone of $A$ one just needs to approximate the effective region of size $\propto \mathcal{O}\left[v_{\mathrm{LR}} t+\log \left(\epsilon^{-1}\right)\right]$. The important point is that this can be done such that the same MPO simulates the light cone of any local operator. For the argument to hold, it is crucial that the MPO of Ref. [39] is a depth-2 quantum circuit, which is impossible in higher dimensions [78].

This MPO can also be implemented in practice [40], following the explicit construction of Ref. [39], which consists on the subsequent application of two local Hamiltonian evolutions. Thus, Result 2 also guarantees an efficient 1D algorithm for short times. The result naturally extends to the simulation of any extensive sum of local operators by linearity.

The exponential scaling in time originates from the error in the Lieb-Robinson bound. For systems with different light cones, the growth in the bond dimension may be much smaller. For instance, many-body localized systems with a "zero velocity" Lieb-Robinson bound $\propto t e^{-l}[79$, 80] can instead be approximated with a bond dimension growing polynomially in time [40].

\section{B. Higher dimensions}

For higher dimensions, we resort to the idea in Sec. III of using partitions of the lattice into hypercubes of length $l_{0}$. The simple approach taken here is to first show that $e^{-i t H} A e^{i t H}$ is close to the evolution of an effective Hamiltonian in the hypercube, and then approximate that effective evolution with a PEPO of small bond dimension. This PEPO can be constructed with the cluster expansion results from Refs. [36,37], adapted to real time evolution (as described in Appendix A 2). The bond dimension of each of these is $D_{l_{0}} \leq \exp \left\{\mathcal{O}\left[|t| \log \left(|t| l_{0}^{d} / \epsilon\right)\right]\right\}$.
The approximation is then accurate if (i) the hypercube is large enough and (ii) $A$ is sufficiently far away from the boundary between hypercubes. However, to construct a scheme that applies to local operators $A$ in any region, we need a tensor network that implements the approximation of a hypercubes conditioned on the support of $A$. This conditioning requires something more involved than just acting with a single PEPO and its adjoint: a tensor map as described in Sec. II. In Appendix C 2 we construct a map that implements a PEPO $M_{p}$ among a possible list as $M_{p} A M_{p}^{\dagger}$, where $M_{p}$ is determined by the region $\mathcal{R}$ that supports $A$. Since the map is linear, it can also act on extensive sums of local operators.

Given that there are $l_{0}^{d}$ possible partitions into hypercubes, we show that this can be done with a tensor network of bond dimension $\mathcal{O}\left(l_{0}^{3 d} D_{l_{0}}^{2}\right)$. Choosing $l_{0}=$ $\tilde{\mathcal{O}}\left[k+v_{\mathrm{LR}} t+\log (1 / \epsilon)\right]$ then yields the following result.

Result 3. There is an explicit construction of a linear PEPO map $\mathcal{M}_{k}^{t}(\cdot)$ such that, for any operator A with support on a small region $\mathcal{R}$, the Heisenberg time evolution is well approximated as

$$
\left\|e^{-i t H} A e^{i t H}-\mathcal{M}_{k}^{t}(A)\right\| \leq 3 \epsilon\|A\| .
$$

If $A$ is a product of Pauli matrices, the map has bond dimension

$$
D \leq \mathcal{O}\left(d|t| \frac{k+v_{L R} t+\log 1 / \epsilon}{\epsilon}\right)^{\mathcal{O}(|t| d)}
$$

whereas for arbitrary $A$ the bond dimension is

$$
D \leq \mathcal{O}\left(d|t| 4^{k} \frac{v_{L R} t+k(1+\log 4 / \epsilon)}{\epsilon}\right)^{\mathcal{O}(|t| d)} .
$$

The proof is shown in Appendix C 2, where we also explain how to construct the tensor network that applies a given PEPO conditioned on the support of the input $A$. This construction of a tensor-network map that acts differently depending on some feature of the input (here, the nontrivial support of the observable) has, as far as we know, not previously appeared in the literature. We believe that this or similar schemes have the potential for further applications.

\section{APPLICATIONS}

Our results apply to a wide range of physical situations. For instance, Result 1 directly shows that it is possible to compute local thermal averages of arbitrary local operators, such as the energy or average magnetization. They can, however, also be used to guarantee approximations of more complex objects. We now elaborate on two of them. 


\section{A. Correlation functions}

A quantity that appears in many relevant situations, mostly pertaining to linear response theory [81], is the two-point correlation function

$$
\langle A(t) A\rangle_{\beta}=\operatorname{Tr}\left[\frac{e^{-\beta H}}{Z} e^{-i t H} A e^{i t H} A\right],
$$

where $A$ is often taken to be an extensive operator. By this, we mean that it has uniform support throughout the lattice as $A=(1 / N) \sum_{x} A_{x} \otimes \mathbb{I}_{\lambda \backslash x}$, where each term has bounded norm $\left\|A_{x}\right\| \leq\|A\|$ and acts on at most $k$ consecutive sites. This is the central object in various areas of quantum dynamics, such as the study of quantum transport, for which $A$ is taken to be a current operator [59] of the relevant conserved quantities.

In the next result, we show how our constructions serve to approximate it. We focus in the one-dimensional case, for which we again assume that Eq. (3) holds with an exponential decay. In higher dimensions, Eq. (12) involves a contraction, which is typically computationally hard $[82,83]$ (although this is potentially not a problem in physically relevant contexts, see Ref. [48]). The result is as follows.

Result 4. The correlation function of an extensive observable $A$ in one dimension can be approximated as

$$
\left|\operatorname{Tr}\left[\tilde{\rho}_{k^{\prime}} M_{k}^{t} A\left(M_{k}^{t}\right)^{\dagger} A\right]-\langle A(t) A\rangle_{\beta}\right| \leq 12 \epsilon\|A\|^{2},
$$

where $k^{\prime}=\mathcal{O}\left[\xi \log (1 / \epsilon)+v_{L R} t+k\right]$, and the operators $\tilde{\rho}_{k^{\prime}}$ and $M_{k}^{t}$ are as defined in Results 1 and 2 .

The proof is shown in Appendix D. In simple terms, Result 4 implies that $\langle A(t) A\rangle_{\beta}$ can well be approximated by a contraction of MPOs of bond dimension at most

$$
D \leq e^{\mathcal{O}(|t|)} \times \text { poly }\left[\frac{k+v_{\mathrm{LR}} t+\xi \log (1 / \epsilon)}{\epsilon}\right] .
$$

See Fig. 3 for an illustration. The biggest drawback of this result is the fast growth in time $t$, which is nevertheless expected in general. This does not necessarily prevent the algorithms from reaching interesting timescales [84], and is likely an overestimation for many important situations at late times. Nevertheless, we believe that this result mathematically justifies the success of previous tensor-network approaches to computing correlation functions [53-59].

\section{B. Quantum quenches}

In quantum quenches, one starts with a pure initial state $|\Phi\rangle$. This is often an easy-to-prepare state, such as a ground state of a gapped model, or a product state. Then, the Hamiltonian is suddenly switched to some arbitrary $H$, and

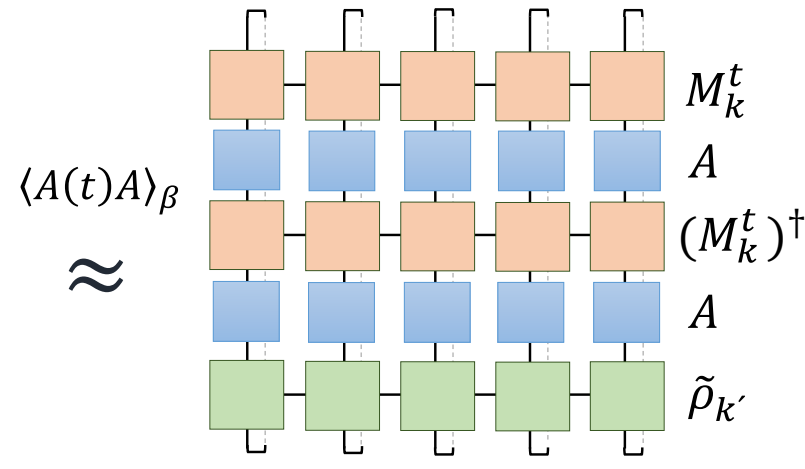

FIG. 3. Schematic illustration of Result 4 with the contraction of tensors involved in approximating the correlation functions $\langle A(t) A\rangle_{\beta}$, where $A$ may be an extensive observable.

the subsequent time evolution is tracked through expectation values of local observables $\langle A(t)\rangle \equiv\langle\Psi|A(t)| \Psi\rangle$. This time evolution can be simulated with the results in Sec. IV, which give an upper bound on the bond dimension required [e.g., Eq. (8)].

This upper bound, however, grows very fast with time, and may become too large at relevant timescales such as the thermalization or Thouless times [85]. This is so even if the local marginals of the evolved state are simply described by a thermal state or a generalized Gibbs ensemble, which depends on very few parameters. In those cases, it is expected that local evolution at late times can also be approximated with a small bond dimension $[60,61,86-88]$.

One of the main results of Ref. [43] (applicable in 1D) can help in this setting: the local marginals of any state $|\Phi(t)\rangle$ on $k$ sites can be approximated with an MPS $|\Psi\rangle$ with bond dimension $D \leq \exp (k / \epsilon)$. One can then potentially simulate $\langle A(t)\rangle$ in $1 \mathrm{D}$ with a bond dimension

$$
D \leq \min \left\{e^{\mathcal{O}(|t|)} \times \text { poly }\left[\frac{k+v_{\mathrm{LR}} t+\log (1 / \epsilon)}{\epsilon}\right], e^{k / \epsilon}\right\} .
$$

It is not clear whether an efficient algorithm to find $|\Psi\rangle$ exists [43]. However, numerical schemes for approximating $\langle A(t)\rangle$ at long times with low bond dimension have already been devised $[60,61]$.

\section{CONCLUSIONS}

We have shown how to construct tensor-network representations of thermal states and time evolution, in a way that local observables are well approximated. This allows us to provably achieve a bond dimension independent of system size in all cases, which contrasts with what is achieved by previous global approximations. These results have implications on the tensor-network simulation of various equilibrium and out of equilibrium situations, and help to mathematically justify the success of previous numerical results. 
Since they hold for any local Hamiltonian, and any timescale and temperature, they are likely not tight in particular cases of interest. For instance, when simulating the long time dynamics of a system that has already thermalized, it seems likely that only a much smaller bond dimension (perhaps independent of time) is required. This intuition is present in previous specific algorithms [60,61].

In all of the proofs, except for Result 2, the constructions involve an average over "local approximations," which we expect to be unnecessary in practice. This also applies to previous results for ground-state approximations [29,42-44]. It would be interesting to find arguments to circumvent this proof idea, perhaps akin to the proof of Result 2 or to other features such as the Markov property of thermal states $[89,90]$. For $1 \mathrm{D}$, this question was dealt with in Ref. [91], where it was shown that there exists a MPO that reproduces the local expectation values of the thermal state with bond dimension $D \leq$ $\exp \tilde{\mathcal{O}}\left[\beta^{2 / 3}+\sqrt{\beta \log (k / \epsilon)}\right]$. This significantly improves on the result of Eq. (5), at the price of not giving an explicit construction of the MPO. It may also be possible to obtain a better higher-dimensional generalization of Result 2 that does not require a tensor map, as Result 3 does.

\section{ACKNOWLEDGMENTS}

The authors acknowledge funding from the Alexander von Humboldt Foundation and from ERC Advanced Grant QUENOCOBA under the EU Horizon 2020 program (Grant Agreement No. 742102) and within the D-ACH Lead-Agency Agreement through Project No. 414325145 (BEYOND C).

\section{APPENDIX A: REVIEW OF THE CLUSTER EXPANSION APPROXIMATION TO THERMAL AND REAL TIME EVOLUTION IN ARBITRARY DIMENSION}

We briefly review the main result of Ref. [36], which provides PEPO approximations for global thermal states in arbitrary spatial dimensions via the cluster expansion [35, 37]. We then explain how an analogous statement holds for the operator $e^{i t H}$.

\section{Thermal state at any temperature}

Let us recall the definitions from Sec. II: $H=\sum_{x} h_{x}$ is a $k$-local Hamiltonian on an arbitrary $d$-dimensional lattice, with up to $K \propto N$ terms, and such that $\max _{x}\left\|h_{x}\right\| \leq h \propto$ $\mathcal{O}(1)$, and the interaction graph has degree at most $z$. We also define the lattice growth constant as $\gamma$.

It was shown in Ref. [35] that there exists an operator $\tilde{\rho}$, defined in terms of the cluster expansion (see Ref. [37] for a detailed proof), which is a good approximation to the thermal state for high temperatures. The statement is as follows. Let $\beta^{*}$ be a constant such that $\gamma e^{(2 z-1) \beta^{*} h}\left(e^{\beta^{*} h}-1\right)<1$ (that is, $\left.\beta^{*} \sim 1 / h d\right)$. If $\beta \leq \beta^{*}$, then

$$
\left\|e^{-\beta H}-\tilde{\rho}\right\|_{1} \leq\left\|e^{-\beta H}\right\|_{1}\left[\exp \left(K \frac{x^{L}}{1-x}\right)-1\right],
$$

where $x \equiv \gamma e^{(2 z-1) \beta h}\left(e^{\beta h}-1\right)<1$. Here, $L$ is a free parameter that determines the size of the clusters in the approximation. Importantly, Eq. (A1) also holds for the norm $\|\cdots\|_{2 M}$ if we change the temperature to $\beta^{\prime}=$ $\beta / 2 M$, such that

$$
\begin{aligned}
& \left\|e^{-(\beta / 2 M) H}-\tilde{\rho}\right\|_{2 M} \\
& \quad \leq\left\|e^{-(\beta / 2 M) H}\right\|_{2 M}\left[\exp \left(K \frac{x^{\prime L}}{1-x^{\prime}}\right)-1\right],
\end{aligned}
$$

with $x^{\prime} \equiv \gamma e^{(2 z-1)(\beta h / 2 M)}\left(e^{\beta h / 2 M}-1\right)<1$. Then, Proposition 1 in Ref. [36] allows us to approximate the thermal state at any temperature.

Proposition 1. If $\epsilon<1 / 3$ and

$$
\left\|e^{-(\beta / 2 M) H}-\tilde{\rho}\right\|_{2 M} \leq \frac{\epsilon}{M}\left\|e^{-(\beta / 2 M) H}\right\|_{2 M},
$$

it follows that

$$
\left\|e^{-\beta H}-\left(\tilde{\rho}^{\dagger} \tilde{\rho}\right)^{2 M}\right\|_{1} \leq \frac{\epsilon}{M}\left\|e^{-\beta H}\right\|_{1} .
$$

To choose an $\epsilon$-close approximation in 1-norm at any temperature, we need to set $M$ large enough such that $\beta / 2 M \leq \beta^{*} \sim 1 / h d$, which amounts to $M=\mathcal{O}(\beta h d)$. Then, for large $L$ the error in Eq. (A2) is

$$
\left[\exp \left(K \frac{x^{\prime L}}{1-x^{\prime}}\right)-1\right] \simeq K x^{\prime L}\left(1-x^{\prime}\right)^{-1}
$$

where $x^{\prime}=\mathcal{O}(1)$. That is, we need to set $L=$ $\mathcal{O}[\log (K M / \epsilon)]=\mathcal{O}[\log (\beta N d / \epsilon)]$.

Moreover, it was shown in Ref. [36] that $\tilde{\rho}$ is a PEPO with bond dimension $D \leq e^{L}$, and thus $\left(\tilde{\rho}^{\dagger} \tilde{\rho}\right)^{2 M}$ has $D \leq$ $e^{2 M L}$. We conclude that to achieve an error $\epsilon$ one requires a bond dimension

$$
D \leq e^{\mathcal{O}[\beta d \log (\beta d N / \epsilon)]} .
$$

\section{Real time evolution}

It can be easily seen that the proof of Eq. (A1) from Ref. [37], and the cluster expansion analysis, also hold for an approximation of $e^{-i t H}$ in operator norm, simply if one substitutes $\beta$ with $|t|$. This just follows from the observation that all the steps in the proof of Ref. [37] remain unchanged if one changes all the norms to operator 
norms, and that there is no further fundamental differences between the operators $e^{-\beta H}$ and $e^{-i t H}$. In the same way, one also has the analog of Proposition 1 for operator norms, which allows us to extend the approximation for arbitrarily long times.

From this observations we conclude that there exists a PEPO $\tilde{U}_{t}$ with bond dimension $D$ such that

$$
\left\|e^{-i t H}-\tilde{U}_{t}\right\| \leq \epsilon
$$

and

$$
D \leq e^{\mathcal{O}\left(|t| d \log \frac{|t| d N}{\epsilon}\right)}
$$

\section{APPENDIX B: LOCAL APPROXIMATIONS TO THERMAL STATES}

Here we show the main result regarding local approximations to thermal states. First, we need the following key assumption for the thermal state $\rho=e^{-\beta H} / Z$.

Definition 1 (Clustering of correlations). The state $\rho$ on a lattice system has $\epsilon(l)$ clustering of correlations if

$$
\max _{X, Y} \frac{\left|\langle X \otimes Y\rangle_{\beta}-\langle X\rangle_{\beta}\langle Y\rangle_{\beta}\right|}{\|X\||| Y||} \leq \varepsilon(l),
$$

where $X$ has support of region $A$ only and $Y$ on region $B$ only, and $l \leq \operatorname{dist}(A, B)$.

The following result on local indistinguishability shows that marginals of thermal states when tracing out a big region are well approximated by the marginal of the thermal state of a much smaller lattice. This is the key ingredient to guarantee the faithfulness of local approximations.

Lemma 1 (Theorem 4, Ref. [49]). Let $H$ be a local bounded Hamiltonian, $\beta$ an inverse temperature and $\rho_{A B}=e^{-\beta H} / \operatorname{Tr}\left[e^{-\beta H}\right]$. Let $A B_{1} B_{2}$ be a separation of the lattice such that $B_{1}$ shields $A$ from $B_{2}$ by a distance of at least l. Let $\rho_{A B_{1}}$ be the Gibbs state on region $A B_{1}$ only (that is, with the terms of the Hamiltonian $H$ that have support on $A B_{1}$ only). If the system has $\epsilon(l)$ clustering of correlations, then

$$
\left\|\operatorname{Tr}_{B}\left[\rho_{A B}\right]-\operatorname{Tr}_{B_{1}}\left[\rho_{A B_{1}}\right]\right\|_{1} \leq C\left|\partial B_{2}\right|\left[\varepsilon(l / 2)+c_{1} e^{-c_{2} l}\right]
$$

where $C>0$ and $c_{1}, c_{2}>0$ are constant and $\left|\partial B_{2}\right|$ is the size of the boundary between $B_{1}$ and $B_{2}$.

This lemma relies on the technique of quantum belief propagation from Ref. [92], from which the contribution $c_{1} e^{-c_{2} l}$ arises. See also Theorem 4 in Ref. [37] for a similar statement at high temperatures only.

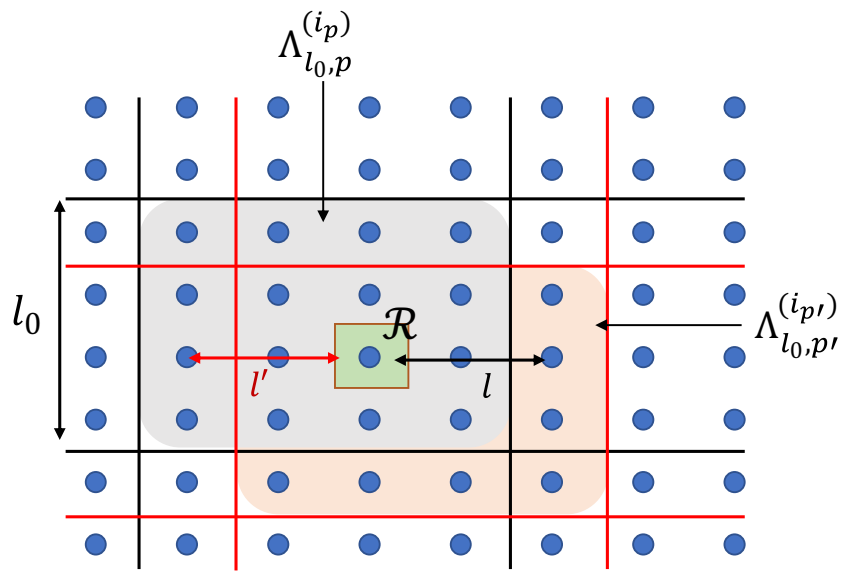

FIG. 4. Schematic illustration of the idea behind the proof of the local approximations to thermal states. $\mathcal{R}$ is our region of interest, and the black and red grids represent two different partitions of the lattice, so that $\mathcal{R}$ is at a distance $l$ and $l^{\prime}$ from each grid. The shaded regions correspond to the hypercubes (in this case, squares) $\Lambda_{l_{0}, p}^{(i)}, \Lambda_{l_{0}, p^{\prime}}^{(i)}$ of size $l_{0}^{d}$ on which $\mathcal{R}$ lies.

To prove the main result of the section, we need to first define the partitions $\mathcal{G}_{p}$ of the $d$-dimensional lattice $\Lambda$ into hypercubes of length $l_{0}$ :

$$
\mathcal{G}_{p}=\left\{\Lambda_{l_{0}, p}^{(i)}: \cup_{i} \Lambda_{l_{0}, p}^{(i)}=\Lambda\right\}
$$

such that $\left|\Lambda_{l_{0}, p}^{(i)}\right| \leq l_{0}^{d}$. The parameter $p$ can, for instance, represent one of the corners of a given hypercube, which defines the position of the boundaries of all the hypercubes, and can adopt $l_{0}^{d}$ values. The hypercubes near the boundaries may have a smaller number of lattice sites. An illustration of these and the other elements of the proof is shown in Fig. 4.

Let $\rho\left(\Lambda_{l_{0}, p}^{(i)}\right)$ be the thermal state with the Hamiltonian containing the terms of $H$ with support only on $\Lambda_{l_{0}, p}^{(i)}$. Each $\rho\left(\Lambda_{l_{0}, p}^{(i)}\right)$ can now be approximated to $\epsilon / 3$ precision in 1-norm by a PEPO $\tilde{\rho}\left(\Lambda_{l_{0}, p}^{(i)}\right)$ of bond dimension $D_{l_{0}}$. In one dimension we have Theorem 3 in Ref. [38], which guarantees

$$
\begin{aligned}
\log D_{l_{0}} \leq & \mathcal{O}\left\{\max \left[\beta, \sqrt{\beta \log \left(\frac{l_{0}}{\epsilon}\right)}\right]\right. \\
& \left.\times \log \left[\beta \log \left(\frac{l_{0}}{\epsilon}\right)\right]\right\},
\end{aligned}
$$

which is subpolynomial in $l_{0}$. In higher dimensions, the result from Ref. [36] described in Appendix A 1 yields $D_{l_{0}} \leq \exp \mathcal{O}\left[\beta d \log \left(\beta d l_{0}^{d} / \epsilon\right)\right]$.

We also need that each of these PEPOs has trace 1 , which can be achieved with a small price in the precision 
as

$$
\begin{aligned}
& \left\|\rho\left(\Lambda_{l_{0}, p}^{(i)}\right)-\frac{\tilde{\rho}\left(\Lambda_{l_{0}, p}^{(i)}\right)}{\operatorname{Tr}\left[\tilde{\rho}\left(\Lambda_{l_{0}, p}^{(i)}\right)\right]}\right\|_{1} \\
& \leq\left\|\rho\left(\Lambda_{l_{0}, p}^{(i)}\right)-\tilde{\rho}\left(\Lambda_{l_{0}, p}^{(i)}\right)\right\|_{1} \\
& \quad+\left\|\tilde{\rho}\left(\Lambda_{l_{0}, p}^{(i)}\right)-\frac{\tilde{\rho}\left(\Lambda_{l_{0}, p}^{(i)}\right)}{\operatorname{Tr}\left[\tilde{\rho}\left(\Lambda_{l_{0}, p}^{(i)}\right)\right]}\right\|_{1} \\
& \leq \frac{1}{3}+\left(1+\frac{\epsilon}{3}\right)\left|\left(1-\frac{1}{\operatorname{Tr}\left[\tilde{\rho}\left(\Lambda_{l_{0}, p}^{(i)}\right)\right]}\right)\right| \leq \epsilon .
\end{aligned}
$$

For simplicity, we assume $\operatorname{Tr}\left[\tilde{\rho}\left(\Lambda_{l_{0}, p}^{(i)}\right)\right]=1$.

Now define the PEPO $\tilde{\rho}_{l_{0}, p}=\bigotimes_{i} \tilde{\rho}\left(\Lambda_{l_{0}, p}^{(i)}\right)$. Our local PEPO approximation is the uniform average over all the partitions of the lattice into hypercubes

$$
\tilde{\rho}=\frac{1}{l_{0}^{d}} \sum_{p} \tilde{\rho}_{l_{0}, p}
$$

which has bond dimension $D \leq D_{l_{0}} \times l_{0}^{d}$. We now show this is a good approximation to the marginal of any region $\mathcal{R} \in \Lambda$ of maximum length $k<l_{0}$. First, we separate the terms in the sum over $p$ by whether $\mathcal{R}$ lies strictly inside one of the hypercubes $\Lambda_{l_{0}, p}^{(i)}$ or not,

$$
\begin{aligned}
& \left\|\operatorname{Tr}_{\Lambda \backslash \mathcal{R}}[\rho]-\operatorname{Tr}_{\Lambda \backslash \mathcal{R}}[\tilde{\rho}]\right\|_{1} \\
& \leq \frac{1}{l_{0}^{d}} \sum_{p}\left\|\operatorname{Tr}_{\Lambda \backslash \mathcal{R}}[\rho]-\operatorname{Tr}_{\Lambda \backslash \mathcal{R}}\left[\tilde{\rho}_{l_{0}, p}\right]\right\|_{1} \\
& \leq \frac{1}{l_{0}^{d}} \sum_{p: \mathcal{R} \nsubseteq \Lambda_{l_{0}, p}^{(i p)}}\left\|\operatorname{Tr}_{\Lambda \backslash \mathcal{R}}[\rho]-\operatorname{Tr}_{\Lambda \backslash \mathcal{R}}\left[\tilde{\rho}_{l_{0}, p}\right]\right\|_{1} \\
& \left.\quad+\sum_{p: \mathcal{R} \subset \Lambda_{l_{0}, p}^{\left(i_{p}\right)}}\left\|\operatorname{Tr}_{\Lambda \backslash \mathcal{R}}[\rho]-\operatorname{Tr}_{\Lambda \backslash \mathcal{R}}\left[\tilde{\rho}_{l_{0}, p}\right]\right\|_{1}\right) \\
& \leq \\
& \quad \frac{1}{l_{0}^{d}}\left(\begin{array}{l}
\left.2 k^{d}+\sum_{p: \mathcal{R} \subset \Lambda_{l_{0}, p}^{(i p)}}\left\|\operatorname{Tr}_{\Lambda \backslash \mathcal{R}}[\rho]-\operatorname{Tr}_{\Lambda \backslash \mathcal{R}}\left[\tilde{\rho}_{l_{0}, p}\right]\right\|_{1}\right)
\end{array}\right)
\end{aligned}
$$

where $i_{p}$ denotes the hypercube $\Lambda_{l_{0}, p}^{\left(i_{p}\right)}$ on which $\mathcal{R}$ lies, for a given $p$ (see Fig. 4). Here, we just use the triangle inequality and the trivial bound $\|\rho-\sigma\|_{1} \leq 2$.
The remaining terms can be bounded with Lemma 1. For a given lattice partition $p$, let $l$ be the minimum distance between $\mathcal{R}$ and the boundary of $\Lambda_{l_{0}, p}^{\left(i_{p}\right)}$. Then,

$$
\begin{aligned}
&\left\|\operatorname{Tr}_{\Lambda \backslash \mathcal{R}}[\rho]-\operatorname{Tr}_{\Lambda \backslash \mathcal{R}}\left[\tilde{\rho}_{l_{0}, p}\right]\right\|_{1} \\
& \leq\left\|\operatorname{Tr}_{\Lambda \backslash \mathcal{R}}[\rho]-\operatorname{Tr}_{\Lambda_{l_{0}, p}^{\left(i_{p}\right)} \backslash \mathcal{R}}\left[\rho\left(\Lambda_{l_{0}, p}^{(i)}\right)\right]\right\|_{1} \\
&+\left\|\operatorname{Tr}_{\Lambda_{l_{0}, p}^{(i p)} \backslash \mathcal{R}}\left[\rho\left(\Lambda_{l_{0}, p}^{(i)}\right)\right]-\operatorname{Tr}_{\Lambda \backslash \mathcal{R}}\left[\tilde{\rho}_{l_{0}, p}\right]\right\|_{1} \\
&= \| \operatorname{Tr}_{\Lambda \backslash \mathcal{R}}[\rho]-\left.\operatorname{Tr}_{\Lambda_{l_{0}, p}^{(i p)} \backslash \mathcal{R}}\left[\rho\left(\Lambda_{l_{0}, p}^{(i)}\right)\right]\right|_{1} \\
&+\left\|\operatorname{Tr}_{\Lambda_{l_{0}, p}^{(i p)} \backslash \mathcal{R}}\left[\rho\left(\Lambda_{l_{0}, p}^{(i)}\right)\right]-\operatorname{Tr}_{\Lambda_{l_{0}, p}^{(i p)} \backslash \mathcal{R}}\left[\tilde{\rho}\left(\Lambda_{l_{0}, p}^{(i)}\right)\right]\right\|_{1} \\
& \leq 2 C d l_{0}^{d-1}\left[\varepsilon(l / 2)+c_{1} e^{-c_{2} l}\right]+\frac{\epsilon}{3}
\end{aligned}
$$

where in the first line we use the triangle inequality, in the second the fact that the PEPOs have trace 1 by assumption, and in the third Lemma 1 (with $\left|\partial B_{2}\right|=2 d l_{0}^{d-1}$ ) and the definition of $\epsilon$. Given that the area of a hypercube of edge $l$ is $2 d l^{d-1}$,

$$
\begin{aligned}
& \left\|\operatorname{Tr}_{\Lambda \backslash \mathcal{R}}[\rho]-\operatorname{Tr}_{\Lambda \backslash \mathcal{R}}[\tilde{\rho}]\right\|_{1} \\
& \leq \frac{2 k^{d}}{l_{0}^{d}}+\frac{\epsilon}{3}+2 C d l_{0}^{-1} \sum_{p: l=\operatorname{dist}\left(\mathcal{R}, \partial \Lambda_{l_{0}, p}^{(i)}\right)}\left[\varepsilon(l / 2)+c_{1} e^{-c_{2} l}\right] \\
& \quad \leq \frac{2 k^{d}}{l_{0}^{d}}+\frac{\epsilon}{3}+4 d^{2} C l_{0}^{-1} \sum_{l=0}^{\left(l_{0}-k\right) / 2} l^{d-1}\left[\varepsilon(l / 2)+c_{1} e^{-c_{2} l}\right] .
\end{aligned}
$$

We now focus on the two different types of decay of the function $\varepsilon(l / 2)$ : exponential and polynomial.

Exponential: In this case $\varepsilon(l / 2) \leq e^{-l / 2 \xi}$. This has been shown in $1 \mathrm{D}$ translation invariant chains [50,67], and it is believed to hold for all $1 \mathrm{D}$ systems $[50,68]$. It has also been shown for higher-dimensional systems at high temperature using the cluster expansion technique in Ref. [37]. There, it is shown that, given $\beta^{*} \equiv \log [(1+\sqrt{1+4 / \gamma}) / 2] / 2 h$, for every $\beta<\beta^{*}$, it holds that

$$
\varepsilon(l) \leq \frac{4 \min \{|\partial A|,|\partial B|\}}{\log 3\left(1-e^{-1 / \xi(\beta)}\right)} e^{-l / \xi(\beta)}
$$

where the correlation length is $\xi(\beta)=\mid\left\{\log \left[\gamma e^{2 \beta h}\right.\right.$ $\left.\left.\left(e^{2 \beta h}-1\right)\right]\right\}^{-1} \mid$. 
Starting from the sum in Eq. (B15), then

$$
\begin{aligned}
& \sum_{l=0}^{\left(l_{0}-k\right) / 2} l^{d-1}\left[\varepsilon(l / 2)+c_{1} e^{-c_{2} l}\right] \\
& \quad \leq\left(c_{1}+1\right) \sum_{l=0}^{\infty} l^{d-1} \exp \left(-\frac{l}{\left(c_{2}+2\right) \xi}\right)=\mathcal{O}\left(\xi^{d}\right)
\end{aligned}
$$

Choosing $l_{0}=C^{\prime} \max \left\{k \epsilon^{-d}, d^{2} \xi^{d} / \epsilon\right\}$ for some constant $C^{\prime}>0$, we obtain $\left\|\operatorname{Tr}_{\Lambda \backslash \mathcal{R}}[\rho]-\operatorname{Tr}_{\Lambda \backslash \mathcal{R}}[\tilde{\rho}]\right\|_{1} \leq \epsilon$. The bond dimension in $1 \mathrm{D}$ is hence

$$
\begin{aligned}
D \leq & \left(\frac{k+\xi}{\epsilon}\right) \times \exp \left(\mathcal { O } \left\{\max \left[\beta, \sqrt{\beta \log \left(\frac{k+\xi}{\epsilon^{2}}\right)}\right]\right.\right. \\
& \left.\left.\times \log \left[\beta \log \left(\frac{k+\xi}{\epsilon^{2}}\right)\right]\right\}\right),
\end{aligned}
$$

which for $\beta=\mathcal{O}(1)$ is quasilinear in $(k+\xi) / \epsilon, D \leq$ $C^{\prime}[(k+\xi) / \epsilon]^{1+o(1)}$. In higher dimensions, it is

$$
D \leq\left(C^{\prime} \beta d \max \left\{\frac{k^{d}}{\epsilon^{2}}, \frac{d^{2 d} \xi^{d^{2}}}{\epsilon^{d+1}}\right\}\right)^{\mathcal{O}(\beta d)},
$$

which is polynomial in $k$ and $\epsilon^{-1}$.

Polynomial: Now assume $\varepsilon(l / 2) \leq R / l^{d+1}$ for any $d$ (note that this assumption is likely not necessary in $1 D$ ). Then the following sum converges:

$$
\begin{aligned}
& \sum_{l=0}^{\left(l_{0}-k\right) / 2} l^{d-1}\left[\varepsilon(l / 2)+c_{1} e^{-c_{2} l}\right] \\
& \quad \leq\left(R+c_{1}\right) \sum_{l=0}^{\infty} l^{d-1} \frac{1}{l^{d+1}}=\mathcal{O}(R)
\end{aligned}
$$

so we simply need to choose $l_{0}=C^{\prime} \max \left\{k \epsilon^{-d}, d R / \epsilon\right\}$ for some constant $C^{\prime}>0$. The resulting bond dimension is thus the same as Eqs. (B18) and (B19) with $\epsilon^{d}$ replaced with $R$.

\section{APPENDIX C: LOCAL APPROXIMATIONS TO TIME EVOLUTION}

Unlike for thermal states, the proofs and statements for one and higher dimensions differ significantly, and are presented separately.

\section{One dimension}

This is based on the main result of Ref. [39], and also relies on the Lieb-Robinson bound [51,52].
Lemma 2 (Lieb-Robinson bound). Let $A$ be a local observable on $k$ sites and $H=\sum_{x} h_{x}$ a uniformly bounded Hamiltonian with finite interaction range. Then there exist constants $c, v_{L R} \geq 0$ such that for all $X$ with $l:=$ $\operatorname{dist}\left(A, X^{c}\right) \geq 2 d-1$ we have

$$
\left\|e^{-i t H} A e^{i t H}-e^{-i t H_{X}} A e^{i t H_{X}}\right\| \leq\|A\| c l^{d-1} e^{v_{L R} t-l},
$$

where $H_{X}$ contains only the terms of $H$ away from $A$ by a distance at most $l$. That is

$$
H_{X}=\sum_{x: s u p p\left(h_{x}\right) \subseteq X} h_{x} .
$$

The result of Ref. [39] is that the time evolution $e^{-i t H}$ on $N$ particles can be approximated with a matrix product unitary $M_{N}^{t}$ of bond dimension $D_{t}$ such that

$$
\left\|e^{-i t H}-M_{N}^{t}\right\| \leq \epsilon
$$

and

$$
D_{t} \leq e^{\mathcal{O}(|t|)+\mathcal{O}[\log (N / \epsilon)]} .
$$

The operator $M_{N}^{t}$ is in fact a quantum circuit of depth 2 , where the gates act on $\left|\Omega_{N}\right| \equiv \mathcal{O}(|t|)+\mathcal{O}[\log (N / \epsilon)]$ adjacent qubits. That is, let $\left\{\Omega_{j}\right\}$ be a partition of the chain into sets of size $\left|\Omega_{N}\right|$, and let $\left\{\Omega_{j}^{\prime}\right\}$ be the same partition, displaced by an amount $\left|\Omega_{N}\right| / 2$. Then

$$
M_{N}^{t}=\left(\bigotimes_{j=1}^{\left\lceil N /\left|\Omega_{N}\right|\right\rceil} U_{\Omega_{j}}(t)\right)\left(\bigotimes_{j=1}^{\left\lceil N /\left|\Omega_{N}\right|\right\rceil} V_{\Omega_{j}^{\prime}}(t)\right)
$$

Our local approximation is $M_{N}^{t}\left(l_{0}\right)$, which is defined in the same way as $M_{N}^{t}$ except for the fact that the partition is into much smaller sets of adjacent sites, each of which has length $\left|\Omega_{l_{0}}\right|=\mathcal{O}(|t|)+\mathcal{O}\left[\log \left(l_{0} / \epsilon\right)\right]$ instead of $\left|\Omega_{N}\right|$. Here $l_{0}$ is a free parameter such that $\left|\Omega_{l_{0}}\right|<l_{0}<N$, otherwise independent of $N$. See Fig. 5 for an illustration of this and the other definitions in the proof.

The goal is to bound the norm $\| e^{-i t H} A e^{i t H}-$ $M_{N}^{t}\left(l_{0}\right) A\left[M_{N}^{t}\left(l_{0}\right)\right]^{\dagger} \|$, for any operator $A$ with support on at most $k$ adjacent sites. First, with the triangle inequality and the Lieb-Robinson bound,

$$
\begin{aligned}
& \left\|e^{-i t H} A e^{i t H}-M_{N}^{t}\left(l_{0}\right) A\left[M_{N}^{t}\left(l_{0}\right)\right]^{\dagger}\right\| \\
& \quad \leq c e^{v_{\mathrm{LR}} t-l}+\left\|e^{-i t H_{A}^{(l)}} A e^{i t H_{A}^{(l)}}-M_{N}^{t}\left(l_{0}\right) A\left[M_{N}^{t}\left(l_{0}\right)\right]^{\dagger}\right\|,
\end{aligned}
$$

where $H_{A}^{(l)}$ is the Hamiltonian containing the terms of $H$ that are a distance $l$ away from $A$, and such that $l_{0}-k-$ $\left|\Omega_{l_{0}}\right| \leq l<l_{0}-k$. 


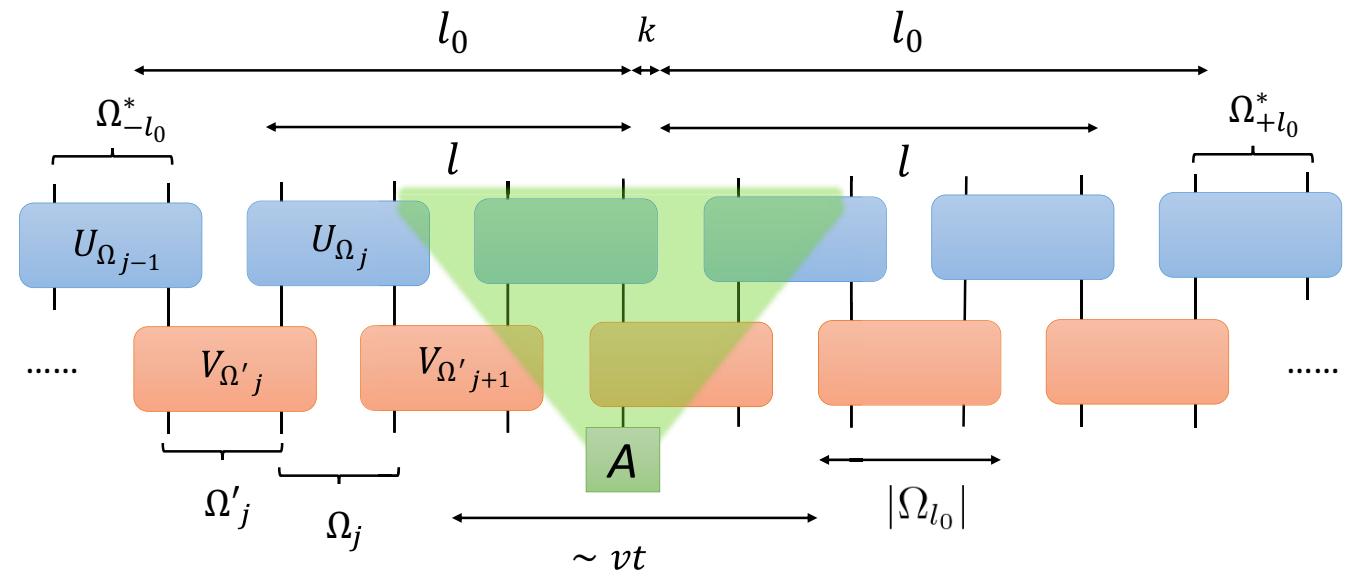

FIG. 5. Schematic illustration of the proof of the local approximation to time evolution. The green area represents the effective Lieb-Robinson light cone of $A$, and the $U_{\Omega_{j}}, V_{\Omega_{j}^{\prime}}$ are the gates on $\left|\Omega_{l_{0}}\right|$ sites that constitute the approximation. A unitary can be applied to regions $\Omega_{ \pm l_{0}}^{*}$ to have the circuit act independently on the region of length $2 l_{0}$, and such that the resulting unitary on that region is an approximation to $e^{-i t H_{A}^{l_{0}}}$, which, when acting on $A$, gives a good approximation to $e^{-i t H} A e^{i t H}$.

Now, let $\Omega_{ \pm l_{0}}^{*}$ be the nearest sets at a distance strictly greater than $l$ from $A$, from the left and the right. The key is to notice that, since $M_{l_{0}}^{t}$ is a depth-2 circuit, there exists a unitary $U_{l_{0}} \equiv U_{-l_{0}} \otimes U_{+l_{0}}$ acting on $\Omega_{ \pm l_{0}}^{*}$ such that $U_{l_{0}} M_{N}^{t}\left(l_{0}\right)=U \otimes M_{l_{0}}^{t}$ for some $U$ with support at distance strictly larger than $l_{0}$ from A (see Fig. 5). By construction, the supports of $U_{l_{0}}$ and $H_{A}^{l}$ do not overlap.

The MPO $M_{l_{0}}^{t}$ is the approximation of the unitary $e^{-i t H_{A}^{\left(l_{0}\right)}}$, on a region of size $\propto l_{0}$, which allows us to use Eq. (C3). That is

$$
\left\|e^{-i t H_{A}^{(l)}} A e^{i t H_{A}^{(l)}}-M_{N}^{t}\left(l_{0}\right) A\left[M_{N}^{t}\left(l_{0}\right)\right]^{\dagger}\right\|
$$
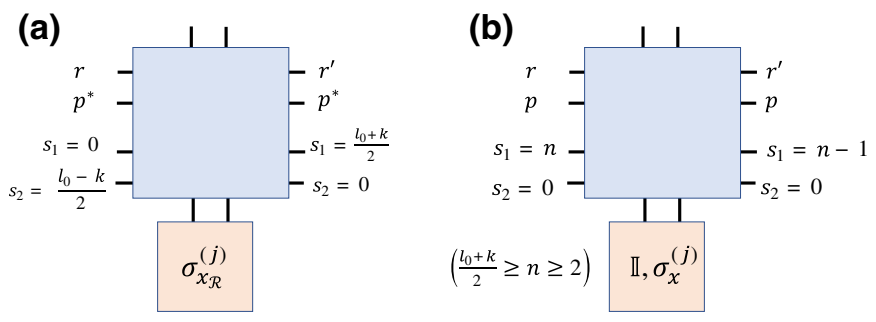

$$
\begin{aligned}
& =\left\|U_{l_{0}} e^{-i t H_{A}^{(l)}} A e^{i t H_{A}^{(l)}} U_{l_{0}}^{\dagger}-U_{l_{0}} M_{N}^{t}\left(l_{0}\right) A\left[M_{N}^{t}\left(l_{0}\right)\right]^{\dagger} U_{l_{0}}^{\dagger}\right\| \\
& =\left\|e^{-i t H_{A}^{(l)}} A e^{i t H_{A}^{(l)}}-\left(U \otimes M_{l_{0}}^{t}\right) A\left(U \otimes M_{l_{0}}^{t}\right)^{\dagger}\right\| \\
& =\left\|e^{-i t H_{A}^{(l)}} A e^{i t H_{A}^{(l)}}-M_{l_{0}}^{t} A\left(M_{l_{0}}^{t}\right)^{\dagger}\right\| \\
& \leq 2\|A\| c e^{v_{\mathrm{LR}} t-l}+\left\|e^{-i t H_{A}^{\left(l_{0}\right)}} A e^{i t H_{A}^{\left(l_{0}\right)}}-M_{l_{0}}^{t} A\left(M_{l_{0}}^{t}\right)^{\dagger}\right\| \\
& \leq 2\|A\| c e^{v_{\mathrm{LR}} t-l}+2 \epsilon\|A\| \\
& \leq 2|| A|| c e^{v_{\mathrm{LR}} t+k+\left|\Omega_{l_{0}}\right|-l_{0}}+2 \epsilon\|A\| \text {. }
\end{aligned}
$$

(d)

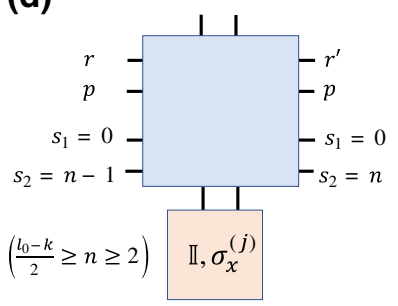

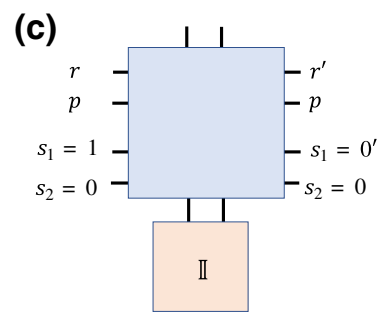

(e)

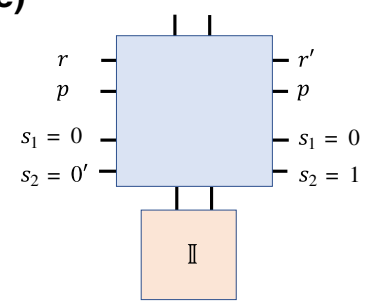

FIG. 6. Tensors: (a) at position $x_{\mathcal{R}}$ indicating the start of region $\mathcal{R}$, fixing $p=p^{*}$ (b) to the right of $x_{\mathcal{R}}$ (c) indicating the right end of the light cone (d) to the left of $x_{\mathcal{R}}$ (e) indicating left end of the light cone. The $s$ index has been split into $\left\{s_{1}, s_{2}\right\}$. 

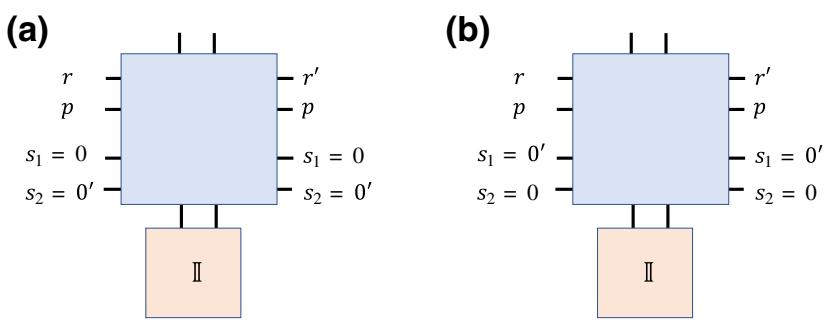

FIG. 7. Tensors: (a) to the left and (b) to the right of the light cone. Their output is the identity.

In the second line we use the unitary invariance of the norm, in the third line the fact that $U_{l_{0}}$ decouples the region at a distance $l$ from the rest, and in the fourth line the Lieb-Robinson bound again, to relate $e^{-i t H_{A}^{(l)}}$ to $e^{-i t H_{A}^{\left(l_{0}\right)}}$.

Thus, since $\left|\Omega_{l_{0}}\right| \ll l_{0}$, if we choose $l_{0}=\mathcal{O}\left[k+v_{\mathrm{LR}} t\right.$ $+\log (1 / \epsilon)]$, we achieve the approximation error $\| e^{-i t H_{A}^{(l)}}$ $A e^{i t H_{A}^{(l)}}-M_{N}^{t}\left(l_{0}\right) A\left[M_{N}^{t}\left(l_{0}\right)\right]^{\dagger}\|\leq 3 \epsilon\| A \|$. Finally, since $M_{N}^{t}\left(l_{0}\right) \equiv M_{k}^{t}$ is by definition a depth-2 circuit with gates acting on $\left|\Omega_{l_{0}}\right|$ sites, the bond dimension required to represent it exactly is

$$
\begin{aligned}
D_{t} & \leq e^{\mathcal{O}(|t|)+\mathcal{O}\left(\log \left\{\left[k+v_{\mathrm{LR}} t+\log (1 / \epsilon)\right] / \epsilon\right\}\right)} \\
& =e^{\mathcal{O}(|t|)} \times \operatorname{poly}\left[\frac{k+v_{\mathrm{LR}} t+\log (1 / \epsilon)}{\epsilon}\right] .
\end{aligned}
$$

\section{Higher dimensions}

For local approximations in higher dimensions, we cannot straightforwardly generalize the proof of the 1D case because we cannot approximate the unitary evolution with a depth-2 circuit, since in higher dimensions one requires depth at least $d+1$ (see Appendix B of Ref. [78]).

Instead, we use an argument similar to that for thermal states in Appendix B. We use the global approximation of $e^{-i t H}$ described in Appendix A to approximate the evolution of the Hamiltonian within the hypercube that has the local operator in the middle. Since this will only work for individual regions, we have to condition the particular partition used on the support of the input, which as we show can be done with a tensor map as defined in Sec. II.

Let us start with the approximation within each of the hypercubes. Lemma 2 guarantees that to simulate the evolution of a local observable $e^{-i t H} A e^{i t H}$ where $\operatorname{supp}(A) \subset \mathcal{R}$ we need only the Hamiltonian of a smaller region $H_{X}$ such that $l:=\operatorname{dist}\left(A, X^{c}\right) \geq 2 d-1$.

We now define the same set of hypercubes covering the lattice as in Appendix B (also see Fig. 4)

$$
\mathcal{G}_{p}=\left\{\Lambda_{l_{0}, p}^{(i)}: \cup_{i} \Lambda_{l_{0}, p}^{(i)}=\Lambda\right\}
$$

such that $\left|\Lambda_{l_{0}, p}^{(i)}\right| \leq l_{0}^{d}$. Again the parameter $p$ defines, e.g., one of the corners of the hypercubes, or any other parameter that determines the whole partition. It can adopt $l_{0}^{d}$ values. To simulate $A(t)$ we then define the PEPO

$$
M_{p}^{\left(l_{0}\right)}=\bigotimes_{i} M_{i, p}^{\left(l_{0}\right)}
$$

where $M_{i, p}^{\left(l_{0}\right)}$ is an $\epsilon$ approximation in operator norm to $e^{-i t H_{i}}$, and $H_{i}=\sum_{x: \operatorname{supp}\left(h_{x}\right) \subseteq \Lambda_{l_{0}, p}^{(i)}} h_{x}$ contains the terms of $H$ with support in $\Lambda_{l_{0}, p}^{(i)}$. Given Eq. (A8), this can be achieved with bond dimension

$$
D_{p} \leq e^{\mathcal{O}\left[|t| d \log \left(|t| d l_{0}^{d} / \epsilon\right)\right]} .
$$

For later convenience we also define the $p=0$ as a special case,

$$
M_{0}^{\left(l_{0}\right)}=\mathbb{I} .
$$

To approximate $A(t)$ we choose the partition $\mathcal{G}_{p}$ so that there exists a hypercube $\Lambda_{l_{0}, p}^{\left(i_{p}\right)}$ such that $\operatorname{dist}\left(A, \backslash \Lambda_{l_{0}, p}^{\left(i_{p}\right)}\right) \geq l=l_{0} / 2-k$ (that is, the region $\mathcal{R}$ is in the middle of the hypercube). This yields an error $\left(2 \epsilon+c l^{d-1} e^{v t-l}\right)\|A\|$, from which we have to then choose $l_{0}=\tilde{\mathcal{O}}\left[k+v_{\mathrm{LR}} t+\log (1 / \epsilon)\right]$ to get $3 \epsilon\|A\|$.

However, we want a PEPO that approximates any A, no matter the region in which it lies. This can be achieved by constructing a linear map $\mathcal{M}_{k}^{t}(A)$ made of tensor contractions such that when applied to $A$ it results on the operator $M_{i, p}^{\left(l_{0}\right)}$ (with the correct partition $p$ and position $i$ ) being applied as $M_{i, p}^{\left(l_{0}\right)} A\left(M_{i, p}^{\left(l_{0}\right)}\right)^{\dagger}$.

For it to be a good approximation of the dynamics, it should be such that the value of $p$ is conditioned on the position of $A$, so that $A$ is in the middle of one of the hypercubes $\Lambda_{l_{0}, p}^{(i)}$. Thus, it should contain the description of the different $M_{p}^{\left(l_{0}\right)}$ (there are $l_{0}^{d}$, one for each value of $p$ ). Importantly, notice that we need to apply a single $M_{i, p}^{\left(l_{0}\right)}$ and not the full $M_{p}^{\left(l_{0}\right)}$. Applying $M_{p}^{\left(l_{0}\right)}$ in every region far from $A$ will incur in additional errors growing with system size, since they are not exactly unitaries and $M_{i, p}^{\left(l_{0}\right)} M_{i, p}^{\left(l_{0}\right) \dagger} \neq \mathbb{I}$. Thus, on top of being able to implement the right partition $p$, we have to make sure that the support beyond the light cone is trivial.

As we now show, all these requirements can be achieved with a bond dimension $D_{p}^{2} \times\left(l_{0}+k\right)^{3 d}$, and so we obtain

$$
D \leq \tilde{\mathcal{O}}\left(d|t| \frac{k+v_{\mathrm{LR}} t+\log 1 / \epsilon}{\epsilon}\right)^{\mathcal{O}(|t| d)}
$$

that is, exponential in $t$ (as expected) and polynomial in $k, \epsilon^{-1}$ now with a degree growing with $t$. 
We now explain how to construct this PEPO map $\mathcal{M}_{k}^{t}(A)$. Let us first limit ourselves to the cases where $A$ is a product of Pauli matrices $A=\bigotimes_{x} \sigma_{x}^{(j)}$ with $j \in\{0,1,2,3\}$, which stand for, respectively, the Pauli $\{\mathbb{I}, X, Y, Z\}$ (the generalization to higher local dimensions is straightforward). By definition the only nonidentity elements in $A$ have support on a small connected region $\mathcal{R}$ of size $k$. The result extends to arbitrary local operators by linearity.

One dimension: We first consider the one-dimensional case, which serves as an illustrative example, and then extend it to larger dimensions. If we simplify the picture by putting the two physical indices of the output and the input together, the tensor network can be drawn schematically as follows:

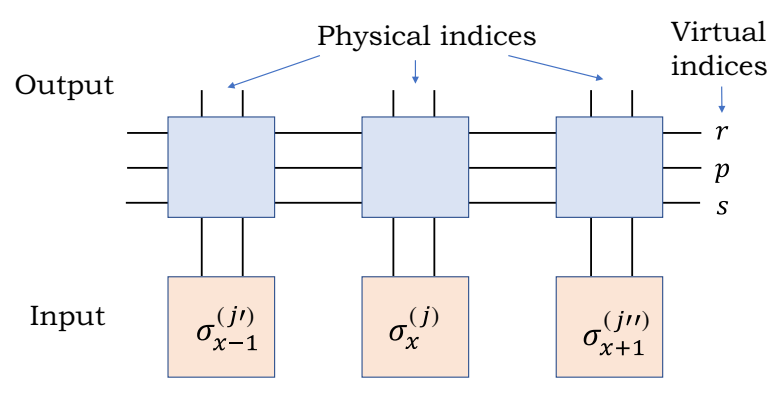

We have three virtual indices with different functions: $p$ is the index that determines the partition into hypercubes from Eq. (C14) to use, and as such is fixed by the position of the nonidentity Pauli matrices in $A$ (i.e., the region $\mathcal{R})$. It can adopt $l_{0}+1$ different values. For a given $p$, index $r$ contains the information about the tensors from the MPO $M_{p}^{\left(l_{0}\right)}$ that are applied to the Pauli, and thus has bond dimension given by Eq. (C16). Finally, $s=\left\{s_{1}, s_{2}\right\}$ are the indices that determine the support of the output MPO, ensuring that it is trivial outside the light cone, as required.

The $r$ index is thus determined by the MPOs $M_{l_{0}}^{\left(l_{0}\right)}$. The $p, s$ indices work as follows: starting from the left of the chain, $s_{1}$ is zero until it reaches a nontrivial Pauli input at site $x_{\mathcal{R}}$. At that point it turns into $\left(l_{0}+k\right) / 2$, and then decreases by 1 at each tensor until it reaches a value we label as $0^{\prime}$, which indicates the end of the light cone at the right. Beyond that point, the desired outcome $\mathcal{M}_{k}^{t}(A)$ must have trivial support, which we enforce by setting the output tensor to be the identity $M_{0}^{\left(l_{0}\right)}=\mathbb{I}$. The point $x_{\mathcal{R}}$ also determines the particular value of $p=p^{*}$, which then sets the PEPO $M_{p^{*}}^{\left(l_{0}\right)}$ to act within the light cone. The index $p^{*}$ is chosen in such a way that the boundary of the hypercubes is $\left(l_{0}-k\right) / 2$ away from the left and $\left(l_{0}+k\right) / 2$ from the right of $x_{\mathcal{R}}$. This sets the right part of the light cone. For the left side of the light cone, we use index $s_{2}$. It starts with value $\left(l_{0}-k\right) / 2$ at $x_{\mathcal{R}}$, and decreases by 1 to the left, while being 0 all the way to the right. The values from $\left(l_{0}-k\right) / 2$ to 1 to the left of $x_{\mathcal{R}}$ allow us to set the output tensor to be that of $M_{p^{*}}^{\left(l_{0}\right)} A\left(M_{p^{*}}^{\left(l_{0}\right)}\right)^{\dagger}$. Then, it goes from 1 to another $0^{\prime}$, after which the output of the tensor is the identity, thus setting the end of the light cone to the right. See Figs 6 and 7 for a schematic illustration of the tensors.

We now illustrate this with the particular tensors. The following combinations of indices are the ones that add to the final result. First, the ones that contribute to the lightcone region are shown in Fig. 6. All of these have as output the tensors corresponding to the MPO $M_{i, p^{*}}^{\left(l_{0}\right)} A\left(M_{i, p^{*}}^{\left(l_{0}\right)}\right)^{\dagger}$ within the light cone. The configuration around $x_{\mathcal{R}}$ can be illustrated as follows:

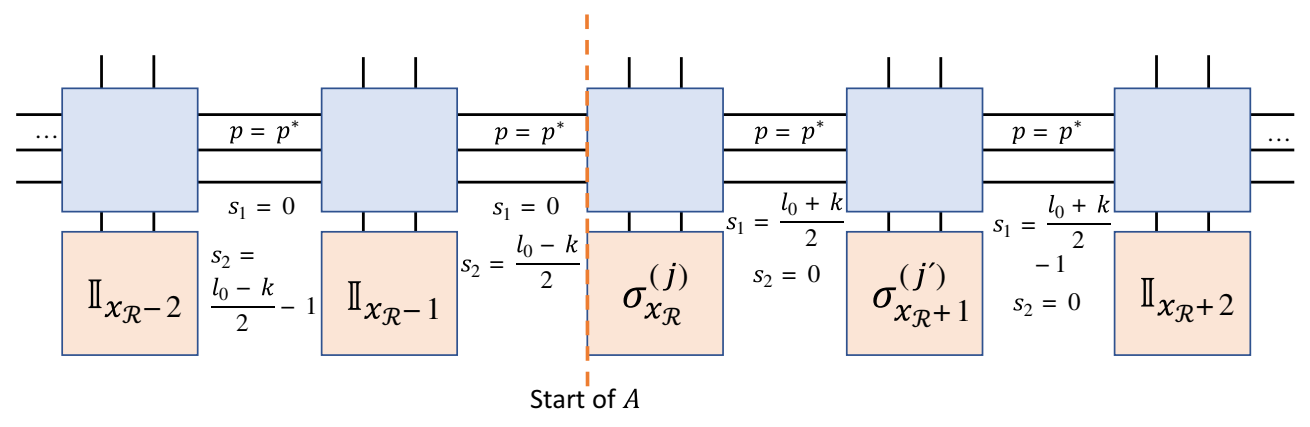

Then, the ones required to set the output beyond the lightcone to be the identity are shown in Fig. 7. To summarize, $p^{*}$ determines the right set of hypercubes, and $s_{1}, s_{2}$ indicates how far we are to the starting point of $\mathcal{R}$, from the right and the left. The tensors apply $M_{p^{*}}^{\left(l_{0}\right)}$ to $A$ [so that the output is $\left.M_{p^{*}}^{\left(l_{0}\right)} A\left(M_{p^{*}}^{\left(l_{0}\right)}\right)^{\dagger}\right]$ unless either $s_{1}, s_{2}=0^{\prime}$, in which case they output II. The result is then $M_{i, p^{*}}^{\left(l_{0}\right)} A\left(M_{i, p^{*}}^{\left(l_{0}\right)}\right)^{\dagger}$ as desired. This is illustrated in Fig. 8.

Higher dimensions: This can be done by establishing the same index configurations as for 1D for every 

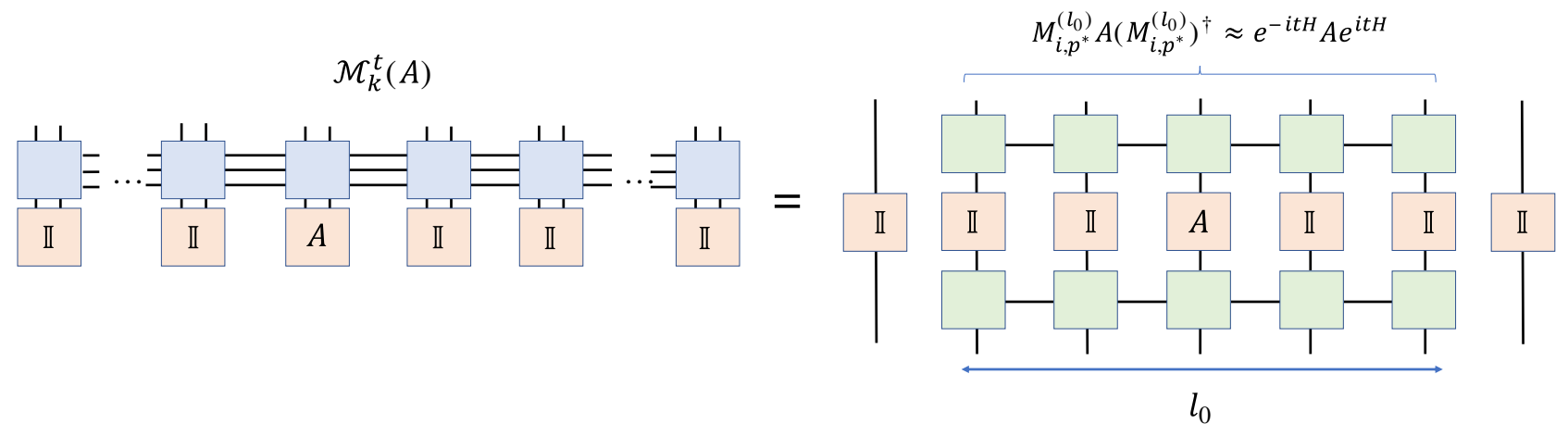

FIG. 8. Final illustration of the scheme in one dimension.

individual direction. Now, we have two $s$ indices $s_{1}^{(q)}, s_{2}^{(q)}$ for each dimension $q \in\{X, Y, \ldots\}$, as well as one index $p^{(q)}$ for each. Let us begin with a particular spatial direction, say $q=X$, with coordinates adopting values $x \in$ $\left\{0,1, \ldots, L_{X}\right\}$ ( $L_{X}$ being the length in that direction). Starting from the first position $x=0$, the index $s_{1}^{X}$ is 0 until any nontrivial Pauli appears at the input, with an $X$ coordinate $x_{\mathcal{R}}$. It is possible that there is more than one nontrivial input with the same initial coordinate $x_{\mathcal{R}}$, but this does not affect the scheme.

At $x_{\mathcal{R}}$, the index $s_{1}^{X}$ is then again changed to $\left(l_{0}+k\right) / 2$, and $s_{2}^{X}$ is changed to $\left(l_{0}-k\right) / 2$. Beyond $x_{\mathcal{R}}, s_{1}^{X}$ decreases along the positive $X$ direction, and $s_{2}^{X}$ decreases along the

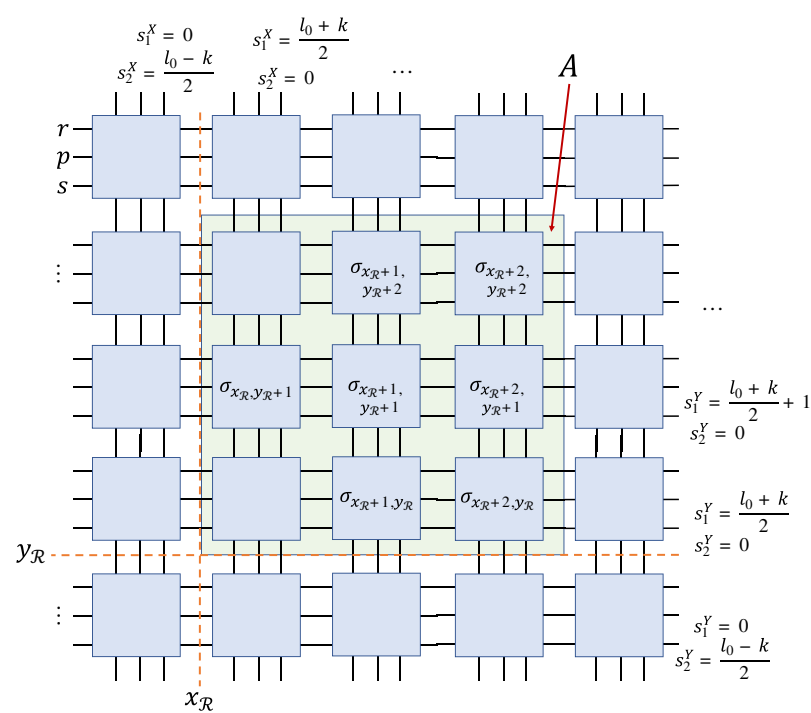

FIG. 9. In the figure, the $\sigma$ represent sites at which the input is not the identity but any other Pauli matrix. The green shaded region is the square region $\mathcal{R}$ in which the observable $A$ has support. The index $s_{1}^{X}$ grows to the right, $s_{2}^{X}$ decreases to the left, and $s_{1}^{Y}, s_{2}^{Y}$, respectively, grow and decrease downwards. $p^{*}$ has two components of $l_{0}+1$ possible values each and is fixed across the entire lattice. We show only virtual indices, and omit the input and output legs of the tensors explicitly for simplicity. negative $X$ direction (while being 0 in the positive direction). When either $s_{1}^{X}=0^{\prime}, s_{2}^{X}=0^{\prime}$, the tensor acts as the identity $M_{0}^{\left(l_{0}\right)}=\mathbb{I}$. Also, the position of that first nontrivial Pauli(s) determines the position of the hypercubes in the $X$ direction, as $p^{X}=p^{X *}$ in the same way as in one dimension. We then repeat the same scheme for every dimension. This is illustrated in 2D in Fig. 9.

With this, we obtain a fixed partition into hypercubes as determined by $p^{*}=\left\{p^{q *}\right\}$, and also a set of indices $s$ whose nonzero value indicate where the output light cone lies. That is, whenever any one of the $2 \times d$ indices $s$ is $0^{\prime}$, we set the local tensor to be that of $M_{0}^{\left(l_{0}\right)}$, and otherwise to be $M_{p^{*}}^{\left(l_{0}\right)}$. This is illustrated in Fig. 10 .

Given this scheme, the map outputs $M_{i, p^{*}}^{\left(l_{0}\right)} A M_{i, p^{*}}^{\left(l_{0}\right) \dagger}$ with the right $\left\{i, p^{*}\right\}$ chosen, such that $\mathcal{R}$ is in the middle of the corresponding hypercube. From our discussion above, this is an $\epsilon$-close approximation to $e^{-i t H} A e^{i t H}$ in operator norm. The whole map involves, on each side of the input $A$, a

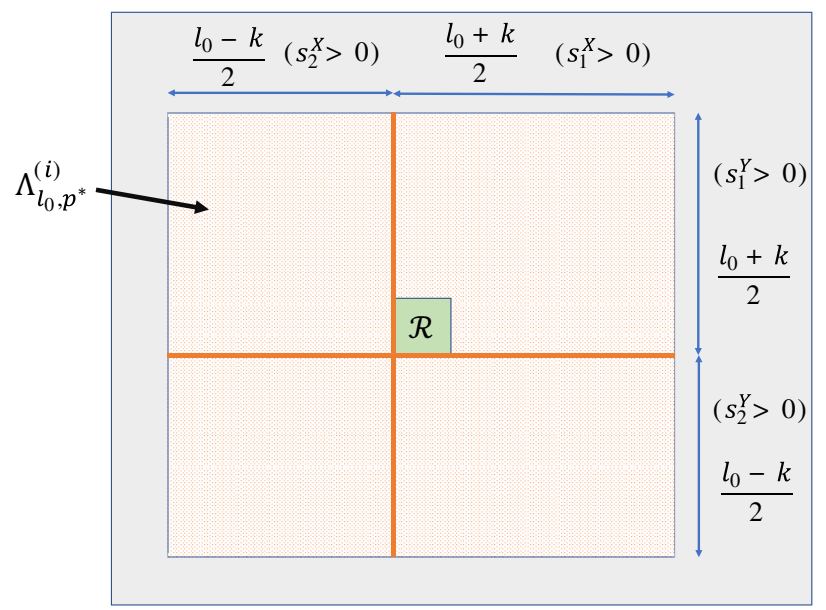

FIG. 10. The orange region represents the hypercube $\Lambda_{l_{0}, p^{*}}^{(i)}$ within the larger lattice in which the output $\mathcal{M}_{k}^{t}(A)=$ $M_{i, p^{*}}^{\left(l_{0}\right)} A M_{i, p^{*}}^{\left(l_{0}\right) \dagger}$ has support. It is determined by the positivity of indices $s_{1,2}^{(q)}$. When any $s_{1,2}^{(q)}=0^{\prime}$, we are outside of the hypercube. 
virtual index $r$ with dimension $D_{p}$, and two additional virtual indices $p$, $\mathbf{s}$ with dimension $\left(l_{0}+1\right)^{d},\left[\left(l_{0}+k\right) / 2\right]^{2 d}$, respectively, to connect the tensors at each site. Thus, the bond dimension can be taken as $D_{p}^{2} \times\left(l_{0}+k\right)^{3 d}$, as stated above.

Finally, our result assumed that $A$ was a product of Paulis. Since the map is linear and any local operator can be written as a linear sum of up to $4^{k}$ Pauli matrices, the result follows by replacing $\epsilon \rightarrow 4^{-k} \epsilon$ in the bound for the bond dimension Eq. (C18).

\section{APPENDIX D: AUTOCORRELATION FUNCTIONS}

We now study how to approximate the correlation functions in one dimension described in the main text

$$
\langle A(t) A\rangle_{\beta}=\operatorname{Tr}\left[\frac{e^{-\beta H}}{Z} e^{-i t H} A e^{i t H} A\right],
$$

with the MPOs from our approximations, as $\operatorname{Tr}\left[\tilde{\rho}_{k^{\prime}} M_{k^{\prime \prime}}^{t}\right.$ $\left.A M_{k^{\prime \prime}}^{t \dagger} A\right]$. Again, $A=(1 / N) \sum_{x} A_{x}$, with $A_{x}$ being supported on $k$ sites and $\left\|A_{x}\right\| \leq\|A\|$. The region sizes $k^{\prime}$ is to be determined, and we choose $k^{\prime \prime}=k$ (the size of the support of $A_{x}$ ). Throughout the proof, there are different sources of $\epsilon$-size errors (coming from Results 1 and 2, and repeated applications of the Lieb-Robinson and the decay of correlations), which we set to be equal.

First, notice that the MPO $M_{k}^{t}$ is a depth-2 quantum circuit with the gates acting on $L=\mathcal{O}(|t|)+$ $\mathcal{O}\left(\log \left\{\left[k+v_{\mathrm{LR}} t+\log \left(\epsilon^{-1}\right)\right] / \epsilon\right\}\right)$ sites. Thus, $M_{k}^{t} A_{x} M_{k}^{t \dagger}$ has support on at most $2 L$ sites. Also, by construction, the MPO $\tilde{\rho}_{k^{\prime}}$ has the following clustering of correlations property, for arbitrary operators $F, G$ of support smaller than $k^{\prime}$ :

$$
\begin{aligned}
& \left|\operatorname{Tr}\left[\tilde{\rho}_{k^{\prime}} F G\right]-\operatorname{Tr}\left[\tilde{\rho}_{k^{\prime}} F\right] \operatorname{Tr}\left[\tilde{\rho}_{k^{\prime}} G\right]\right|=0 \\
& \quad \text { if } \operatorname{dist}(F, G)>\mathcal{O}\left(\frac{k^{\prime}}{\epsilon}\right), \\
& \left|\operatorname{Tr}\left[\tilde{\rho}_{k^{\prime}} F G\right]-\operatorname{Tr}\left[\tilde{\rho}_{k^{\prime}} F\right] \operatorname{Tr}\left[\tilde{\rho}_{k^{\prime}} G\right]\right| \\
& \leq\left(2 \epsilon+K e^{-\operatorname{dist}(F, G) / \xi}\right)\|F\|\|G\| \\
& \quad \text { if } \operatorname{dist}(F, G) \leq \mathcal{O}\left(\frac{k^{\prime}}{\epsilon}\right),
\end{aligned}
$$

where $\epsilon$ here comes from the error in the approximation in Result 1 . The first line is due to the fact that $\tilde{\rho}_{k^{\prime}}$ is constructed as a mixture of partitions, which are products over distances larger than $\mathcal{O}\left(k^{\prime} / \epsilon\right)$. The second is due to the fact that, when the observables are close (and within the same cell in the partition), one recovers the correlation length $\xi$ in the thermal state from Eq. (3), with an additional error $2 \epsilon$.
Let us look now at the pairs $A_{x}, A_{y}$ that are far away in the lattice. The previous equations imply that

$$
\begin{aligned}
& \left|\operatorname{Tr}\left[\tilde{\rho}_{k^{\prime}} M_{k}^{t} A_{x} M_{k}^{\dagger \dagger} A_{y}\right]-\operatorname{Tr}\left[\tilde{\rho}_{k^{\prime}} M_{k}^{t} A_{x} M_{k}^{t \dagger}\right] \operatorname{Tr}\left[\tilde{\rho}_{k^{\prime}} A_{y}\right]\right| \\
& \leq 3 \epsilon\|A\|^{2} \quad \text { if } \operatorname{dist}(x, y)>\mathcal{O}\left(\frac{k^{\prime}}{\epsilon}\right) \\
& \quad+\mathcal{O}\left(v_{\mathrm{LR}} t+k+\log 1 / \epsilon\right),
\end{aligned}
$$

which follows from Eq. (D2). Also, from Eq. (D3)

$$
\begin{aligned}
& \left|\operatorname{Tr}\left[\tilde{\rho}_{k^{\prime}} M_{k}^{t} A_{x} M_{k}^{t \dagger} A_{y}\right]-\operatorname{Tr}\left[\tilde{\rho}_{k^{\prime}} M_{k}^{t} A_{x} M_{k}^{t \dagger}\right] \operatorname{Tr}\left[\tilde{\rho}_{k^{\prime}} A_{y}\right]\right| \\
& \leq 3 \epsilon\|A\|^{2} \quad \text { if } \operatorname{dist}(x, y)>\mathcal{O}\left(\xi \log \frac{1}{\epsilon}\right) \\
& \quad+\mathcal{O}\left(v_{\mathrm{LR}} t+k+\log 1 / \epsilon\right) .
\end{aligned}
$$

Thus, for a good approximation to these terms it suffices to constrain $k^{\prime} \geq 2 L$, in which case

$$
\begin{aligned}
& \left|\operatorname{Tr}\left[\tilde{\rho}_{k^{\prime}} M_{k}^{t} A_{x} M_{k}^{\dagger \dagger} A_{y}\right]-\left\langle M_{k}^{t} A_{x} M_{k}^{\dagger \dagger}\right\rangle_{\beta}\left\langle A_{y}\right\rangle_{\beta}\right| \\
& \leq 7 \epsilon\|A\|^{2} \quad \text { if } \operatorname{dist}(x, y)>\mathcal{O}\left(\xi \log \frac{1}{\epsilon}\right) \\
& \quad+\mathcal{O}\left(v_{\mathrm{LR}} t+k+\log 1 / \epsilon\right),
\end{aligned}
$$

where the 7 comes from repeated applications of Result 1 . This thus includes all the $x, y$ pairs with

$$
\begin{aligned}
\operatorname{dist}(x, y)> & \mathcal{O}\left(\min \left\{\xi \log \frac{1}{\epsilon}, \frac{k^{\prime}}{\epsilon}\right\}\right) \\
& +\mathcal{O}\left(v_{\mathrm{LR}} t+k+\log 1 / \epsilon\right) .
\end{aligned}
$$

Using Result 2, we have that $\mid\left\langle M_{k}^{t} A_{x} M_{k}^{t \dagger}\right\rangle_{\beta}\left\langle A_{y}\right\rangle_{\beta}-$ $\left\langle A_{x}(t)\right\rangle_{\beta}\left\langle A_{y}\right\rangle_{\beta} \mid \leq 3 \epsilon\|A\|^{2}$. Repeated applications of the Lieb-Robinson bound yield $\left|\left\langle A_{x}(t)\right\rangle_{\beta}\left\langle A_{y}\right\rangle_{\beta}-\left\langle A_{x}(t) A_{y}\right\rangle_{\beta}\right|$ $\leq 2 \epsilon\|A\|^{2}$, and so we obtain

$$
\left|\operatorname{Tr}\left[\tilde{\rho}_{k^{\prime}} M_{k}^{t} A_{x} M_{k}^{\dagger \dagger} A_{y}\right]-\left\langle A_{x}(t) A_{y}\right\rangle_{\beta}\right| \leq 12 \epsilon\|A\|^{2} .
$$

For the pairs that are nearby, let us now constrain further $k^{\prime} \geq \xi+2 L$. This means that, given Eq. (D7) we still need to cover the pairs $x, y$ such that

$$
\operatorname{dist}(x, y) \leq \mathcal{O}\left(v_{\mathrm{LR}} t+k+\xi \log 1 / \epsilon\right) .
$$

This can clearly be done by choosing $k^{\prime}=\mathcal{O}\left[\left(v_{\mathrm{LR}} t+k+\right.\right.$ $\xi) \log 1 / \epsilon]$, which is consistent with $k^{\prime} \geq \xi+2 L$. Then, Result 1 implies that

$$
\left|\operatorname{Tr}\left[\tilde{\rho}_{k^{\prime}} M_{k}^{t} A_{x} M_{k}^{t \dagger} A_{y}\right]-\left\langle M_{k}^{t} A_{x} M_{k}^{i \dagger} A_{y}\right\rangle_{\beta}\right| \leq \epsilon\|A\|^{2},
$$

and from Result 2, $\left|\left\langle M_{k}^{t} A_{x} M_{k}^{t \dagger} A_{y}\right\rangle_{\beta}-\left\langle e^{-i t H} A_{x} e^{i t H} A_{y}\right\rangle_{\beta}\right| \leq$ $3 \epsilon\|A\|^{2}$. This thus shows that Eq. (D8) holds for all pairs 
$x, y$ regardless of their distance. The final result follows by approximating each term in the sum $\left\langle e^{-i t H} A e^{i t H} A\right\rangle_{\beta}=$ $\left(1 / N^{2}\right) \sum x, y\left\langle e^{-i t H} A_{x} e^{i t H} A_{y}\right\rangle_{\beta}$.

[1] S. R. White, Density Matrix Formulation for Quantum Renormalization Groups, Phys. Rev. Lett. 69, 2863 (1992).

[2] U. Schollwöck, The density-matrix renormalization group in the age of matrix product states, Ann. Phys. (N. Y.) 326, 96 (2011).

[3] F. Verstraete, J. J. Garcöa-Ripoll, and J. I. Cirac, Matrix Product Density Operators: Simulation of FiniteTemperature and Dissipative Systems, Phys. Rev. Lett. 93, 207204 (2004).

[4] R. Orús and G. Vidal, Infinite time-evolving block decimation algorithm beyond unitary evolution, Phys. Rev. B 78, 155117 (2008).

[5] S. R. White, Minimally Entangled Typical Quantum States at Finite Temperature, Phys. Rev. Lett. 102, 190601 (2009).

[6] E. M. Stoudenmire and S. R. White, Minimally entangled typical thermal state algorithms, New J. Phys. 12, 055026 (2010).

[7] W. Li, S.-J. Ran, S.-S. Gong, Y. Zhao, B. Xi, F. Ye, and G. $\mathrm{Su}$, Linearized Tensor Renormalization Group Algorithm for the Calculation of Thermodynamic Properties of Quantum Lattice Models, Phys. Rev. Lett. 106, 127202 (2011).

[8] M. Binder and T. Barthel, Minimally entangled typical thermal states versus matrix product purifications for the simulation of equilibrium states and time evolution, Phys. Rev. B 92, 125119 (2015).

[9] P. Czarnik and J. Dziarmaga, Variational approach to projected entangled pair states at finite temperature, Phys. Rev. B 92, 035152 (2015).

[10] B.-B. Chen, Y.-J. Liu, Z. Chen, and W. Li, Series-expansion thermal tensor network approach for quantum lattice models, Phys. Rev. B 95, 161104(R) (2017).

[11] B.-B. Chen, L. Chen, Z. Chen, W. Li, and A. Weichselbaum, Exponential Thermal Tensor Network Approach for Quantum Lattice Models, Phys. Rev. X 8, 031082 (2018).

[12] A. Kshetrimayum, M. Rizzi, J. Eisert, and R. Orús, Tensor Network Annealing Algorithm for Two-Dimensional Thermal States, Phys. Rev. Lett. 122, 070502 (2019).

[13] C.-M. Chung and U. Schollwöck, Minimally entangled typical thermal states with auxiliary matrix-product-state bases, ArXiv:1910.03329 (2019).

[14] A. J. Daley, C. Kollath, U. Schollwöck, and G. Vidal, Timedependent density-matrix renormalization-group using adaptive effective Hilbert spaces, J. Stat. Mech.: Theory Exp. 2004, P04005 (2004).

[15] A. E. Feiguin and S. R. White, Time-step targeting methods for real-time dynamics using the density matrix renormalization group, Phys. Rev. B 72, 020404 (2005).

[16] S. R. Manmana, in AIP Conference Proceedings (AIP, 2005).

[17] J. J. García-Ripoll, Time evolution of matrix product states, New J. Phys. 8, 305 (2006).
[18] G. Vidal, Classical Simulation of Infinite-Size Quantum Lattice Systems in One Spatial Dimension, Phys. Rev. Lett. 98, 070201 (2007).

[19] J. Haegeman, J. I. Cirac, T. J. Osborne, I. Pižorn, H. Verschelde, and F. Verstraete, Time-Dependent Variational Principle for Quantum Lattices, Phys. Rev. Lett. 107, 070601 (2011).

[20] M. L. Wall and L. D. Carr, Out-of-equilibrium dynamics with matrix product states, New J. Phys. 14, 125015 (2012).

[21] C. Karrasch, J. E. Moore, and F. Heidrich-Meisner, Realtime and real-space spin and energy dynamics in onedimensional spin-1/2 systems induced by local quantum quenches at finite temperatures, Phys. Rev. B 89, 075139 (2014).

[22] M. P. Zaletel, R. S. K. Mong, C. Karrasch, J. E. Moore, and F. Pollmann, Time-evolving a matrix product state with long-ranged interactions, Phys. Rev. B 91, 165112 (2015).

[23] J. Haegeman, C. Lubich, I. Oseledets, B. Vandereycken, and F. Verstraete, Unifying time evolution and optimization with matrix product states, Phys. Rev. B 94, 165116 (2016).

[24] E. Ronca, Z. Li, C. A. Jimenez-Hoyos, and G. K.-L. Chan, Time-step targeting time-dependent and dynamical density matrix renormalization group algorithms with $a b$ initio Hamiltonians, J. Chem. Theory Comput. 13, 5560 (2017).

[25] S. Paeckel, T. Köhler, A. Swoboda, S. R. Manmana, U. Schollwöck, and C. Hubig, Time-evolution methods for matrix-product states, Ann. Phys. (N. Y.) 411, 167998 (2019).

[26] B. Vanhecke, L. Vanderstraeten, and F. Verstraete, Symmetric cluster expansions with tensor networks, Phys. Rev. A 103, 1020402 (2021).

[27] M. B. Hastings, An area law for one-dimensional quantum systems, J. Stat. Mech.: Theory Exp. 2007, P08024 (2007).

[28] I. Arad, A. Kitaev, Z. Landau, and U. Vazirani, An area law and sub-exponential algorithm for 1D systems, ArXiv:1301.1162 (2013).

[29] Y. Huang, Area law in one dimension: Degenerate ground states and Renyi entanglement entropy, ArXiv:1403.0327 (2015).

[30] Z. Landau, U. Vazirani, and T. Vidick, A polynomial time algorithm for the ground state of one-dimensional gapped local Hamiltonians, Nat. Phys. 11, 566 (2015).

[31] Y. Huang, A polynomial-time algorithm for the ground state of one-dimensional gapped Hamiltonians, ArXiv: 1406.6355 (2015).

[32] C. T. Chubb and S. T. Flammia, Computing the degenerate ground space of gapped spin chains in polynomial time, Chicago J. Theor. Comput. Sci. 22, 1 (2016).

[33] N. D. Beaudrap, T. J. Osborne, and J. Eisert, Ground states of unfrustrated spin Hamiltonians satisfy an area law, New J. Phys. 12, 095007 (2010).

[34] A. Anshu, I. Arad, and D. Gosset, An area law for 2D frustration-free spin systems, ArXiv:2103.02492 (2021).

[35] M. B. Hastings, Solving gapped Hamiltonians locally, Phys. Rev. B 73, 085115 (2006). 
[36] A. Molnar, N. Schuch, F. Verstraete, and J. I. Cirac, Approximating Gibbs states of local Hamiltonians efficiently with projected entangled pair states, Phys. Rev. B 91, 045138 (2015).

[37] M. Kliesch, C. Gogolin, M. Kastoryano, A. Riera, and J. Eisert, Locality of Temperature, Phys. Rev. X 4, 031019 (2014).

[38] T. Kuwahara, A. M. Alhambra, and A. Anshu, Improved Thermal Area Law and Quasilinear Time Algorithm for Quantum Gibbs States, Phys. Rev. X 11, 011047 (2021).

[39] T. J. Osborne, Efficient Approximation of the Dynamics of One-Dimensional Quantum Spin Systems, Phys. Rev. Lett. 97, 157202 (2006).

[40] M. B. Hastings, Observations outside the light cone: Algorithms for nonequilibrium and thermal states, Phys. Rev. B 77, 144302 (2008).

[41] Y. Huang, Computing energy density in one dimension, ArXiv:1505.00772 (2015).

[42] N. Schuch and F. Verstraete, Matrix product state approximations for infinite systems, ArXiv:1711.06559 (2017).

[43] A. M. Dalzell and F. G. S. L. Brandão, Locally accurate MPS approximations for ground states of one-dimensional gapped local Hamiltonians, Quantum 3, 187 (2019).

[44] Y. Huang, Approximating local properties by tensor network states with constant bond dimension, ArXiv:1903.10048 (2019).

[45] Y. Huang, Computing local properties in the trivial phase, ArXiv:2001.10763 (2020).

[46] Y. Huang, Matrix product state approximations: Bringing theory closer to practice, Quantum Views 3, 26 (2019).

[47] S. Michalakis and J. P. Zwolak, Stability of frustration-free Hamiltonians, Commun. Math. Phys. 322, 277 (2013).

[48] M. Schwarz, O. Buerschaper, and J. Eisert, Approximating local observables on projected entangled pair states, Phys. Rev. A 95, 060102 (2017).

[49] F. G. S. L. Brandão and M. J. Kastoryano, Finite correlation length implies efficient preparation of quantum thermal states, Commun. Math. Phys. 365, 1 (2019).

[50] A. Bluhm, Ángela Capel, and A. Pérez-Hernández, Exponential decay of mutual information for Gibbs states of local Hamiltonians, ArXiv:2104.04419 (2021).

[51] E. H. Lieb and D. W. Robinson, in Statistical Mechanics (Springer, Berlin, Heidelberg, 1972), p. 425.

[52] S. Bravyi, M. B. Hastings, and F. Verstraete, Lieb-Robinson Bounds and the Generation of Correlations and Topological Quantum Order, Phys. Rev. Lett. 97, 050401 (2006).

[53] T. Barthel, U. Schollwöck, and S. R. White, Spectral functions in one-dimensional quantum systems at finite temperature using the density matrix renormalization group, Phys. Rev. B 79, 245101 (2009).

[54] C. Karrasch, J. H. Bardarson, and J. E. Moore, FiniteTemperature Dynamical Density Matrix Renormalization Group and the Drude Weight of Spin-1/2 Chains, Phys. Rev. Lett. 108, 227206 (2012).

[55] C. Karrasch, J. H. Bardarson, and J. E. Moore, Reducing the numerical effort of finite-temperature density matrix renormalization group calculations, New J. Phys. 15, 083031 (2013).
[56] T. Barthel, Precise evaluation of thermal response functions by optimized density matrix renormalization group schemes, New J. Phys. 15, 073010 (2013).

[57] A. C. Tiegel, S. R. Manmana, T. Pruschke, and A. Honecker, Matrix product state formulation of frequencyspace dynamics at finite temperatures, Phys. Rev. B 90, 060406 (2014).

[58] C. Karrasch, D. M. Kennes, and F. Heidrich-Meisner, Spin and thermal conductivity of quantum spin chains and ladders, Phys. Rev. B 91, 115130 (2015).

[59] B. Bertini, F. Heidrich-Meisner, C. Karrasch, T. Prosen, R. Steinigeweg, and M. Znidaric, Finite-temperature transport in one-dimensional quantum lattice models, ArXiv:2003.03334 (2020).

[60] C. D. White, M. Zaletel, R. S. K. Mong, and G. Refael, Quantum dynamics of thermalizing systems, Phys. Rev. B 97, 035127 (2018).

[61] E. Leviatan, F. Pollmann, J. H. Bardarson, D. A. Huse, and E. Altman, Quantum thermalization dynamics with matrixproduct states, ArXiv:1702.08894 (2017).

[62] B. Kloss, Y. B. Lev, and D. Reichman, Time-dependent variational principle in matrix-product state manifolds: Pitfalls and potential, Phys. Rev. B 97, 024307 (2018).

[63] A. Gilchrist, N. K. Langford, and M. A. Nielsen, Distance measures to compare real and ideal quantum processes, Phys. Rev. A 71, 062310 (2005).

[64] B. Pirvu, V. Murg, J. I. Cirac, and F. Verstraete, Matrix product operator representations, New J. Phys. 12, 025012 (2010).

[65] M. Zwolak and G. Vidal, Mixed-State Dynamics in One-Dimensional Quantum Lattice Systems: A TimeDependent Superoperator Renormalization Algorithm, Phys. Rev. Lett. 93, 207205 (2004).

[66] F. Verstraete and J. I. Cirac, arXiv:0407066 (2004).

[67] H. Araki, Gibbs states of a one dimensional quantum lattice, Commun. Math. Phys. 14, 120 (1969).

[68] A. W. Harrow, S. Mehraban, and M. Soleimanifar, in Proceedings of the 52nd Annual ACM SIGACT Symposium on Theory of Computing (ACM, 2020).

[69] J. Fröhlich and D. Ueltschi, Some properties of correlations of quantum lattice systems in thermal equilibrium, J. Math. Phys. 56, 053302 (2015).

[70] M. Hartmann, Minimal length scales for the existence of local temperature, Contemp. Phys. 47, 89 (2006).

[71] A. Ferraro, A. Garcöa-Saez, and A. Acön, Intensive temperature and quantum correlations for refined quantum measurements, EPL 98, 10009 (2012).

[72] S. Hernández-Santana, A. Riera, K. V. Hovhannisyan, M. Perarnau-Llobet, L. Tagliacozzo, and A. Acín, Locality of temperature in spin chains, New J. Phys. 17, 085007 (2015).

[73] S. Hernández-Santana, A. Molnar, C. Gogolin, J. I. Cirac, and A. Acín, Locality of temperature and correlations in the presence of non-zero-temperature phase transitions, ArXiv:2010.15256 (2020).

[74] M. Rigol, T. Bryant, and R. R. P. Singh, Numerical LinkedCluster Approach to Quantum Lattice Models, Phys. Rev. Lett. 97, 187202 (2006).

[75] S. Bravyi, Upper bounds on entangling rates of bipartite Hamiltonians, Phys. Rev. A 76, 052319 (2007). 
[76] J. Eisert and T. J. Osborne, General Entanglement Scaling Laws from Time Evolution, Phys. Rev. Lett. 97, 150404 (2006).

[77] M. Mariën, K. M. Audenaert, K. Van Acoleyen, and F. Verstraete, entanglement rates and the stability of the area law for the entanglement entropy, Commun. Math. Phys. 346, 35 (2016).

[78] J. Haah, M. B. Hastings, R. Kothari, and G. H. Low, Quantum algorithm for simulating real time evolution of lattice Hamiltonians, SIAM J. Comput., FOCS18-250 (2021).

[79] C. K. Burrell and T. J. Osborne, Bounds on the Speed of Information Propagation in Disordered Quantum Spin Chains, Phys. Rev. Lett. 99, 167201 (2007).

[80] E. Hamza, R. Sims, and G. Stolz, Dynamical localization in disordered quantum spin systems, Commun. Math. Phys. 315, 215 (2012).

[81] R. Kubo, Statistical-mechanical theory of irreversible processes. I. General theory and simple applications to magnetic and conduction problems, J. Phys. Soc. Jpn. 12, 570 (1957).

[82] N. Schuch, M. M. Wolf, F. Verstraete, and J. I. Cirac, Computational Complexity of Projected Entangled Pair States, Phys. Rev. Lett. 98, 140506 (2007).

[83] J. Haferkamp, D. Hangleiter, J. Eisert, and M. Gluza, Contracting projected entangled pair states is average-case hard, Phys. Rev. Res. 2, 013010 (2020).
[84] A. M. Alhambra, J. Riddell, and L. P. García-Pintos, Time Evolution of Correlation Functions in Quantum ManyBody Systems, Phys. Rev. Lett. 124, 110605 (2020).

[85] L. D'Alessio, Y. Kafri, A. Polkovnikov, and M. Rigol, From quantum chaos and eigenstate thermalization to statistical mechanics and thermodynamics, Adv. Phys. 65, 239 (2016).

[86] M. Berta, F. G. S. L. Brandão, J. Haegeman, V. B. Scholz, and F. Verstraete, Thermal states as convex combinations of matrix product states, Phys. Rev. B 98, 235154 (2018).

[87] S. Xu and B. Swingle, Accessing scrambling using matrix product operators, Nat. Phys. 16, 199 (2019).

[88] A. Hallam, J. G. Morley, and A. G. Green, The Lyapunov spectra of quantum thermalisation, Nat. Commun. 10, 2708 (2019).

[89] K. Kato and F. G. S. L. Brandão, Quantum approximate Markov chains are thermal, Commun. Math. Phys. 370, 117 (2019).

[90] T. Kuwahara, K. Kato, and F. G. Brandão, Clustering of Conditional Mutual Information for Quantum Gibbs States above a Threshold Temperature, Phys. Rev. Lett. 124, 220601 (2020).

[91] Y. Huang, Locally accurate matrix product approximation to thermal states, ArXiv:2106.03854 (2021).

[92] M. B. Hastings, Quantum belief propagation: An algorithm for thermal quantum systems, Phys. Rev. B 76, 201102 (2007). 